\title{
THE NONSTANDARD THEORY OF TOPOLOGICAL VECTOR SPACES
}

\author{
C. WARD HENSON AND L. C. MOORE, JR.
}

\begin{abstract}
In this paper the nonstandard theory of topological vector spaces is developed, with three main objectives: (1) creation of the basic nonstandard concepts and tools; (2) use of these tools to give nonstandard treatments of some major standard theorems; (3) construction of the nonstandard hull of an arbitrary topological vector space, and the beginning of the study of the class of spaces which results.
\end{abstract}

Introduction. Let $\pi$ be a set theoretical structure and let $* \pi$ be an enlargement of $\pi$. Let $(E, \theta)$ be a topological vector space in $M . ~ \$ \$ 1$ and 2 of this paper are devoted to the elementary nonstandard theory of $(E, \theta)$. In particular, in $\$ 1$ the concept of $\theta$-finiteness for elements of ${ }^{*} E$ is introduced and the nonstandard hull of $(E, \theta)$ (relative to $* \mathbb{M}$ ) is defined. $\$ 2$ introduces the concept of $\theta$-boundedness for elements of ${ }^{*} E$. In $\$ 5$ the elementary nonstandard theory of locally convex spaces is developed by investigating the mapping in $* \pi$ which corresponds to a given pairing.

In $\$ \$ 6$ and 7 we make use of this theory by providing nonstandard treatments of two aspects of the existing standard theory. In $\$ 6$, Luxemburg's characterization of the pre-nearstandard elements of ${ }^{*} E$ for a normed space $(E, \rho)$ is extended to Hausdorff locally convex spaces $(E, \theta)$. This characterization is used to prove the theorem of Grothendieck which gives a criterion for the completeness of a Hausdorff locally convex space. In $\$ 7$ a nonstandard proof is given of the theorem of Krein which states that, in a complete Hausdorff locally convex space, the weakly closed convex hull of a weakly compact set is again weakly compact. The nonstandard proof is somewhat simpler than the standard ones. In particular, we give a quite simple proof of the preliminary result (Corollary 7.3) which states that if $A$ is a $\theta$-compact set in a topological vector space $(E, \theta)$ and if $\left\{f_{n}\right\}$ is a sequence of $\theta$-continuous linear functionals on $E$ which is uniformly bounded on $A$ and which converges to 0 on $A$, pointwise, then the sequence $\left\{f_{n}\right\}$ converges to 0 , pointwise, on the closed convex hull of $A$.

$\S 8$ concerns the natural pairing between the nonstandard hull $(\hat{E}, \hat{\rho})$ of a normed space $(E, \rho)$ and the nonstandard hull $\left(\widehat{E^{\prime}}, \widehat{\rho^{\prime}}\right)$ of the dual space $\left(E^{\prime}, \rho^{\prime}\right)$. We give a standard condition on $(E, \rho)$ (independent of $* \Re$ ) which is equivalent to each of

Presented in part to the Society, March 31, 1972 under the title The dual space of the nonstandard hull of a normed space; received by the editors October 18, 1971.

AMS (MOS) subject classifications (1970). Primary 02H25, 46Axx; Secondary 26A98, $54 \mathrm{~J} 05$. 
the following statements, provided that $* \mathbb{N}$ is $\boldsymbol{N}_{1}$-saturated:

(i) the dual space of $(\hat{E}, \hat{\rho})$ is $\left(\widehat{E^{\prime}}, \widehat{\rho^{\prime}}\right)$;

(ii) $(\hat{E}, \hat{\rho})$ is reflexive.

It is a consequence of this result that there is a reflexive Banach space whose nonstandard hull is a nonreflexive Banach space whenever $* \pi$ is $\boldsymbol{N}_{1}$-saturated. On the other hand, we show that, under the same saturation assumptions, if $1<p<\infty$ and $1 / p+1 / q=1$, then $\hat{l}_{p}$ and $\hat{L}_{p}([0,1])$ are reflexive spaces whose dual spaces are $\hat{l}_{q}$ and $\hat{L}_{q}([0,1])$ respectively.

In $\$ 4$ we discuss the extent to which the nonstandard hull of an arbitrary Hausdorff topological vector space $(E, \theta)$ in $\mathbb{N}$ is independent of the enlargement $* \pi$. It is shown that either there are nonstandard hulls of $(E, \theta)$ which have arbitrarily large cardinality or every nonstandard hull of $(E, \theta)$ is isomorphic to the completion of $(E, \theta)$. In the former case, every nonstandard hull of $(E, \theta)$ contains the completion of $(E, \theta)$ as a proper subspace.

In $\$ 3$ the concept of $\theta$-finiteness, introduced in $\$ 1$, is compared with the concept of finiteness with respect to the unique translation invariant uniformity on $E$ which generates the topology $\theta$. The latter concept of finiteness was introduced for a general uniform space in [3].

Preliminaries. Throughout this paper $\mathbb{N}$ will denote a set theoretical structure and $* \pi$ will denote an enlargement of $M$. The basic framework of nonstandard analysis used here can be found in [6] and [9], where $\pi$ and $* \pi$ are structures for type theory. We assume that $M$ has as elements the sets $N$ (of positive integers), $R$ (of real numbers) and $C$ (of complex numbers). Moreover, the embedding $x \mapsto \mapsto^{*}$ of $\mathbb{M}$ into $* \pi$ is assumed to be the identity on $N, R$ and $C$. As usual, the extensions to ${ }^{*} C$ of the algebraic operations,$+ \cdot$ and || on $C$ are denoted by the same symbols. The same is true of the extension to ${ }^{*} R$ of the ordering $<$ on $R$.

For $p, q \in{ }^{*} C, p={ }_{1} q$ means that $p-q$ is infinitesimal. If $p$ is a finite element of ${ }^{*} C$, then the standard part of $p$ in $C$ is denoted by st $(p)$. Define subsets ${ }^{*} C_{0}$ and ${ }^{*} C_{1}$ of ${ }^{*} C$ by

$$
\begin{aligned}
& { }^{*} C_{0}=\left\{p \mid p \in{ }^{*} C \text { and } p \text { is finite }\right\} \\
& { }^{*} C_{1}=\left\{p \mid p \in{ }^{*} C \text { and } p \text { is infinitesimal }\right\} .
\end{aligned}
$$

Also define ${ }^{*} R_{0}={ }^{*} R \cap{ }^{*} C_{0}$ and ${ }^{*} R_{1}={ }^{*} R \cap{ }^{*} C_{1}$ (so that ${ }^{*} R_{0}$ is the set of finite elements of ${ }^{*} R$ and ${ }^{*} R_{1}$ is the set of infinitesimal elements of ${ }^{*} R$ ). By $R^{+}$we denote the set of $\lambda$ in $R$ with $\lambda \geq 0$.

Let $X$ be any set in $\mathbb{M}$. We define $*[X]$ to be the set of standard elements of $* X$; that is,

$$
{ }^{*}[X]=\left\{{ }^{*} x \mid x \in X\right\} .
$$

If $p$ is an element of $* X$, then $\mathrm{Fil}_{X}(p)$ is the ultrafilter on $X$ determined by $p$ : 


$$
\operatorname{Fil}_{X}(p)=\left\{Y \mid Y \subset X \text { and } . p \epsilon^{*} Y\right\}
$$

$\left(\mathrm{Fil}_{X}(p)\right.$ is written simply as $\mathrm{Fil}(p)$ when convenient.) If $\mathcal{F}$ is any collection of subsets of $X$ which has the finite intersection property, then the filter monad of $\mathcal{F}$, $\mu(\mathcal{F})$, is defined by

$$
\mu(\mathcal{F})=\bigcap\left\{{ }^{*} A \mid A \in \mathcal{F}\right\}=\bigcap^{*}[\mathcal{F}] .
$$

Recall that if $* M$ is an enlargement of $M$, then for each $X$ in $M$ and each coliection $\mathcal{F}$ of subsets of $X$ which has the finite intersection property, $\mu(\mathcal{F}) \neq \emptyset$. In fact, in that case there is an element $A$ of $* \mathcal{F}$ which satisfies $A \subset \mu(\mathcal{F})$. (Theorem 2.1.5(a) of [6].)

Although we usually assume only that $* \pi$ is an enlargement of $M$, it is occasionally necessary, in order to achieve a smooth theory, to assume that $* \pi$ is also $\kappa$-saturated (in the sense of [6]) for a sufficiently large cardinal number $\kappa$. This assumption will always be stated explicitly where needed. (When discussing the property of $\kappa$-saturation, we will always assume that $\kappa$ is uncountable.) Recall that $* M$ is $\kappa$-saturated if and only if for every $X$ in $M$ and every collection $\mathbb{Q}$ of internal subsets of $* X$, if $\mathfrak{Q}$ has the finite intersection property and has cardinality less than $\kappa$, then $\mathbb{Q}$ has nonempty intersection. (Theorem 2.7.12 of [6].)

Another useful property of $* M$, related to $\kappa$-saturation, we choose to call $\kappa$ enlarging ( $\kappa$ an infinite cardinal). We say that $* \pi$ is a $\kappa$-enlarging extension of $\pi$ if and only if for every $X$ in $\mathbb{N}$ and every collection $\mathfrak{A}$ of internal subsets of ${ }^{*} X$, if $\mathbb{Q}$ has the finite intersection property and the number of elements of $\mathfrak{Q}$ which are not standard is less than $\kappa$, then $\mathfrak{Q}$ has nonempty intersection. (Recall that a subset of ${ }^{*} X$ is standard if it equals ${ }^{*} Y$ for some subset $Y$ of $X$.) We will be primarily interested in the property of being an $\boldsymbol{N}_{0}$-enlarging extension of $\mathbb{M}$. This property of * $M$ is equivalent to asserting that the three (equivalent) conditions in Theorem 2.7.3 of [6] hold in * $\pi$.

Note that if $\kappa$ is a cardinal, then the direct limit of a chain (of order type $\kappa$ ) of successive enlargements, beginning with $\mathbb{M}$, is a $k$-enlarging extension of $\mathbb{M}$. Also, if $\kappa$ is larger than the cardinality of every set in $M$, then $* \pi$ is $\kappa$-saturated if and only if $* \mathbb{N}$ is $\kappa$-enlarging. Therefore, each structure $\mathbb{N}$ has $\kappa$-saturated extensions and $\kappa$-enlarging extensions for every cardinal number $\kappa$.

Throughout this paper $E$ and $F$ will denote vector spaces over $R$ or $C$, usually assumed to be members of $\pi$. For convenience we let $K$ stand for either $R$ or $C$. Thus ${ }^{*} K_{0}$ is the set of finite elements of ${ }^{*} K$ and ${ }^{*} K_{1}$ is the set of infinitesimal elements of ${ }^{*} K$. If $E$ is a $K$-vector space, then the addition on ${ }^{*} E$ is denoted by $+($ as it is in $E)$ and the scalar multiplication operation on ${ }^{*} K \times{ }^{*} E$ takes $(\lambda, p)$ to $\lambda p$.

Now let $E$ be a $K$-vector space. The algebraic dual of $E$ (consisting of all linear functionals from $E$ to $K$ ) is denoted by $E^{\#}$. If $\theta$ is a vector topology on $E$, 
then the dual space of $(E, \theta)$ (consisting of all $\theta$-continuous members of $\left.E^{\#}\right)$ is denoted by $(E, \theta)^{\prime}$, or simply by $E^{\prime}$.

If $E$ and $F$ are $K$-vector spaces, then a pairing of $E$ and $F$ is a bilinear functional $\langle\ldots, \ldots\rangle$ on $E \times F$ which satisfies:

(i) if $x \in E$ and $x \neq 0$, then $\langle x, y\rangle \neq 0$ for some $y \in F$, and

(ii) if $y \in F$ and $y \neq 0$, then $\langle x, y\rangle \neq 0$ for some $x \in E$.

We denote the weak topology on $E$ defined by $F$ and the given pairing by $\sigma(E, F)$. Also, $\tau(E, F)$ is the Mackey topology and $\beta(E, F)$ is the strong topology determined by the pairing.

Let $E$ be a $K$-vector space which is in $\pi$ and let $\theta$ be a vector topology on $E$. Denote by $\mathcal{U}(\theta)$ the filter of $\theta$-neighborhoods of 0 , and let $\mathcal{O}(\theta)$ be the unique translation invariant uniformity on $E$ generating the topology $\theta$. Then $\vartheta(\theta)$ is the filter on $E \times E$ generated by the filter base of all sets of the form

$$
\{(x, y) \mid x, y \in E \text { and } x-y \in U\}
$$

where $U$ ranges over $\mathcal{U}(\theta)$.

Recall that for each $x$ in $E, \mu_{\theta}(x)$ is defined by

$$
\mu_{\theta}(x)=\mu\left(\mathcal{F}_{x}\right)
$$

where $\mathcal{F}_{x}$ is the filter of $\theta$-neighborhoods of $x$. Since $\mathfrak{F}_{x}=\{x+U \mid U \in \mathcal{U}(\theta)\}$ it follows that $\mu_{\theta}(x)={ }^{*} x+\mu_{\theta}(0)$ for every $x$ in $E$. Also, the monad of the filter $\mathcal{O}(\theta)$ is an equivalence relation on ${ }^{*} E$. For each $p$ in ${ }^{*} E$ we denote the equivalence class of $p$ under this relation by $\mu(p)$. Note that the definition of $\mathcal{O}(\theta)$ implies that $q \in \mu(p) \Leftrightarrow p-q \in \epsilon^{*} U$ for all $U$ in $U(\theta)$. Therefore, $\mu(p)=p+\mu_{\theta}(0)$ for every $p$ in ${ }^{*} E$. In particular, this shows that if $x$ is in $E$, then $\mu(* x)=\mu_{\theta}(x)$. Note that since $\mathcal{U}(\theta)$ is the filter of neighborhoods of 0 for a vector topology on $E$, it follows that $\mu_{\theta}(0)$ is closed under addition and under multiplication by elements of ${ }^{*} K_{0}$.

Recall that an element $p$ of ${ }^{*} E$ is $\theta$-nearstandard if $p \in \mu_{\theta}(x)$ for some $x$ in $E$. The set of $\theta$-nearstandard elements of ${ }^{*} E$ will be denoted by $\mathrm{ns}_{\theta}\left({ }^{*} E\right)$, or simply by $n{ }_{\theta}$. We say that an element $p$ of ${ }^{*} E$ is $\theta$-pre-nearstandard if it is $\mathcal{O}(\theta)$-prenearstandard in the sense of [6]. The set of $\theta$-pre-nearstandard elements of ${ }^{*} E$ will be denoted by pns ${ }_{\theta}\left({ }^{*} E\right)$, or simply by pns $\theta$. Thus $p$ is in pns $\theta$ if and only if there is a $\theta$-Cauchy filter $\mathcal{F}$ on $E$ which satisfies $\mu(\mathcal{F}) \subset \mu(p)$.

We define an important topology $\tilde{\theta}$ on ${ }^{*} E$ as follows: For each $p$ in ${ }^{*} E$ let $\mathcal{F}_{p}$ be the filter on ${ }^{*} E$ generated by the filter base $\left\{p+{ }^{*} U \mid U \in \mathcal{U}(\theta)\right\}$. It can be verified that the filter system $\left\{\mathcal{F}_{p} \mid p \in{ }^{*} E\right\}$ satisfies the conditions which insure that there exists a topology $\tilde{\theta}$ on ${ }^{*} E$ such that $\mathcal{F}_{p}$ is the filter of $\tilde{\theta}$-neighborhoods of $p$ for each $p$ in ${ }^{*} E$. Note that the definition of the filters $\mathcal{F}_{p}$ insures that $\tilde{\theta}$ is a group topology on the additive group of ${ }^{*} E$. For each $x$ in $E, \mathcal{F}_{\left({ }^{*} x\right)}$ is the 
filter on ${ }^{*} E$ generated by $*\left[\mathcal{F}_{x}\right]$, where $\mathcal{F}_{x}$ is the filter of $\theta$-neighborhoods of $x$ in $E$. Thus the mapping $x \mapsto^{*} x$ is a homeomorphism of $(E, \theta)$ into $(* E, \widetilde{\theta})$.

There is another convenient procedure for describing $\tilde{\theta}$. As is discussed in [3], there is a natural uniformity $\vartheta(\theta)$ on ${ }^{*} E$ which is the filter on ${ }^{*} E \times{ }^{*} E$ generated by the filter base $*[\vartheta(\theta)]$. The topology $\tilde{\theta}$ is then the topology defined on ${ }^{*} E$ by the uniformity $O(\theta)$.

Finally, we let $\kappa(\theta)$ denote the smallest infinite cardinal number $\kappa$ such that there is a local base $\mathcal{U}$ at 0 for $\theta$ with the cardinality of $\mathcal{U}$ equal to $\kappa$. ( $\mathcal{U}$ is a local base at 0 for $\theta$ if $\mathcal{U}$ is a filter base which generates the filter $\mathcal{U}(\theta)$.) Let $\kappa(\theta)^{+}$be the smallest cardinal number strictly greater than $\kappa(\theta)$. The degree of saturation required on $* \pi$ in order to achieve a satisfactory nonstandard theory of the space $(E, \theta)$ seems to be $\kappa(\theta){ }^{+}$-saturation.

1. $\theta$-finite elements of $* E$; the nonstandard hull. Let $E$ be a vector space over $K$ which is an element of $\pi$ and let $\theta$ be a vector topology on $E$.

Definition 1.l. An element $p$ of ${ }^{*} E$ is $\theta$-finite if, for each $\theta$-neighborhood $U$ of 0 , there exists an integer $n$ in $N$ which satisfies $p \in n^{*} U$.

The set of $\theta$-finite elements of ${ }^{*} E$ will be denoted by fin ${ }^{(* E)}$, or simply by fin $\theta$.

Remark. Suppose that the vector topology $\theta$ is defined on $E$ by a norm $\rho$. If $U$ is the closed unit ball of $(E, \rho)$, then ${ }^{*} U=\left\{p \mid p \in \epsilon^{*} E\right.$ and $\left.{ }^{*} \rho(p) \leq 1\right\}$. Thus an element $p$ of ${ }^{*} E$ is $\theta$-finite if and only if $p \in n^{*} U$ for some $n$ in $N$; that is, $p$ is $\theta$-finite if and only if ${ }^{*} \rho(p)$ is finite. Thus $f_{\theta}$ consists of the elements of ${ }^{*} E$ which are norm-finite in the sense of [6]. We will often write fin $\rho$ in place of fin $_{\theta}$ when $\theta$ is defined by a norm $\rho$.

Theorem 1.2. (i) fin ${ }_{\theta}\left({ }^{*} E\right)$ is closed under addition and under multiplication by elements of ${ }^{*} K_{0}$.

(ii) The topology $\tilde{\theta}$ restricted to $\operatorname{fin}_{\ell}\left({ }^{*} E\right)$ is a vector topology and $\operatorname{fin}_{\theta}\left({ }^{*} E\right)$ is the largest $K$-subspace of ${ }^{*} E$ on which $\tilde{\theta}$ induces a vector topology.

(iii) fin ${ }_{\theta}(* E)$ is $\tilde{\theta}$-closed.

(iv) $\operatorname{pns}_{\theta}\left({ }^{*} E\right)$ is the $\tilde{\theta}$-closure of $*[E]$ and is a subset of $\operatorname{fin}_{\theta}\left({ }^{*} E\right)$.

Proof. (i) is immediate. To prove (ii) recall that $\tilde{\theta}$ is a group topology on the additive group of ${ }^{*} E$ and has the set $\{* U \mid U$ is a circled $\theta$-neighborhood of 0$\}$ as a local base at 0 . Therefore $\tilde{\theta}$ defines a vector topology on a $K$-subspace $F$ of ${ }^{*} E$ if and only if each of the sets ${ }^{*} U \cap F$ is absorbing. ( $U$ a circled $\theta$-neighborhood of 0.) Obviously fin $\theta$ is the largest $K$-subspace of ${ }^{*} E$ for which this is true.

(iii) Given any $p$ in ${ }^{*} E$ which is not $\theta$-finite, there is a $\theta$-neighborhood $U$ of 0 such that $p \notin n^{*} U$ for every $n$ in $N$. It follows that $p+{ }^{*} U$ is a $\tilde{\theta}$-neighborhood of $p$ which is disjoint from fin $\theta$. This shows that fin $\theta$ is $\tilde{\theta}$-closed.

(iv) Theorem 3.15 .2 of [6] implies that pns ${ }_{\theta}$ is the $\tilde{\theta}$-closure of $*[E]$ in ${ }^{*} E$. 
(The extra assumptions made on $* \pi$ in [6] are not needed for this result.) Since it is evident that $*[E]$ is a subset of fin $_{\theta}$, (iv) follows directly from (iii).

Theorem 1.3. An element $p$ of ${ }^{*} E$ is $\theta$-finite if and only if $\lambda_{p} \in \mu_{\theta}(0)$ whenever $\lambda \in{ }^{*} K_{1}$.

Proof. If $\lambda p \in \mu_{\theta}(0)$ for every $\lambda$ in ${ }^{*} K_{1}$ and if $U$ is a $\theta$-neighborhood of 0 , then the internal set $\left\{\omega \mid \omega \epsilon^{*} N\right.$ and $\left.p \in \omega^{*} U\right\}$ must contain a standard integer. Therefore the condition implies that $p$ is $\theta$-finite.

Conversely, suppose $p$ is $\theta$-finite. Let $U$ be a circled $\theta$-neighborhood of 0 . Then $p \in n^{*} U$ for some $n$ in $N$. Therefore, $p \in \omega^{*} U$ for every infinite $\omega$ in ${ }^{*} N$. But given $\lambda \in \epsilon^{*} K_{1}$ with $\lambda \neq 0$, there exists an infinite $\omega$ in ${ }^{*} N$ such that $1 \geq \lambda \omega$. Therefore, $\lambda p \in \lambda \omega^{*} U \subset{ }^{*} U$. It follows that $\lambda p$ is in $\mu_{\theta}(0)$ whenever $\lambda$ is in ${ }^{*} K_{1}$.

Remark. Since $\mu_{\theta}(0)$ is closed under addition and under multiplication by elements of $* K_{0}$, it is an immediate consequence of Theorem 1.3 that

$$
p \in \operatorname{fin}_{\theta} \Rightarrow \mu(p) \subset \operatorname{fin}_{\theta} \text {. }
$$

Also, Theorem 1.3 implies that $p$ is $\theta$-finite if and only if

$$
\omega \epsilon^{*} N \sim N \Rightarrow(1 / \omega) p \dot{\epsilon} \mu_{\theta}(0)
$$

Theorem 1.4. If $\mathcal{F}$ is a filter on $E$, then $\mu(\mathcal{F}) \subset$ fin $_{\theta}\left({ }^{*} E\right)$ if and only if for every $\theta$-neighborbood $U$ of 0 there exists $n \in N$ which satisfies $n U \in \mathfrak{F}$.

Proof. Let $\mathcal{F}$ be a filter on $E$ which satisfies the latter condition. Given a $\theta$ neighborhood $U$ of 0 , there exists $n \in N$ which satisfies $\mu(\mathcal{F}) \subset n^{*} U$. It follows directly that every element of $\mu(\mathcal{F})$ is $\theta$-finite.

Conversely, suppose $\mathcal{F}$ is a filter on $E$ and $\mu(\mathfrak{F})$ is contained in fin $\theta$. If $\mathfrak{F}$ fails to satisfy the condition, then there is a $\theta$-neighborhood $U$ of 0 which satisfies $n U \notin \mathscr{F}$ for every $n$ in $N$. We may assume that $U$ is circled, so that $U \subset 2 U$ $C$... It follows that the collection $\mathcal{F} \cup\{E \sim n U \mid n \in N\}$ has the finite intersection property. Since $* \pi$ is an enlargement, this implies that there exists $p \in \epsilon^{*} E$ which satisfies $p \in \mu(\mathfrak{F})$ and $p \in *(E \sim n U)$ for every $n$ in $N$; that is, $p \notin n^{*} U$ for every $n$ in $N$. It follows that $p$ is not $\theta$-finite, which is a contradiction.

Remark. It is an immediate consequence of Theorem 1.4 that, for each $p$ in $* E$,

$$
p \in \operatorname{fin}_{\theta} \leftrightarrow \mu(\operatorname{Fil}(p)) \subset \operatorname{fin}_{\theta} .
$$

Corollary 1.5. If $\theta$ and $\theta^{\prime}$ are vector topologies on $E$, then

(i) $\theta \subset \theta^{\prime} \leftrightarrow$ fin $_{\theta} \supset$ fin $\theta^{\prime}$,

(ii) $\theta=\theta^{\prime} \leftrightarrow$ fin $_{\theta}=$ fin $_{\theta^{\prime}}$.

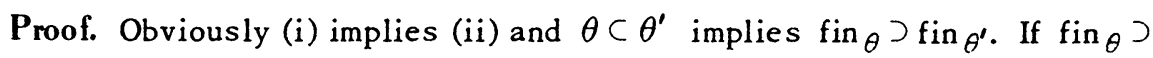
fin $\theta^{\prime}$, then $\mu_{\theta^{\prime}}(0) \subset$ fin $\theta$. It follows from Theorem 1.4 that for each $\theta$-neighborhood 
$U$ of 0 there is an integer $n$ in $N$ such that $n U$ is a $\theta^{\prime}$-neighborhood of 0 . This implies $\theta \subset \theta^{\prime}$.

Theorem 1.6. Assume $* M$ is $\kappa(\theta)^{+}$-saturated. Then for each $p$ in $\mu_{\theta}(0)$ there exists an infinite integer $\omega$ in $* N$ which satisfies $\omega p \in \mu_{\theta}(0)$.

Proof. Let $p$ be in $\mu_{\theta}(0)$ and let $\mathcal{U}$ be a local base at 0 for $\theta$ such that $\mathcal{U}$ has cardinality $\kappa(\theta)$. For each $n \in N$ and $U \in \mathcal{U}$ define the internal set $A(n, . U)$ by

$$
A(n, U)=\left\{m \mid m \epsilon^{*} N, n<m \text { and } m p \epsilon^{*} U\right\}
$$

Since $\mu_{\theta}(0)$ is closed under multiplication by elements of $N$, each set $A(n, U)$ is nonempty. It follows that the collection of sets $A(n, U)$ is a collection of internal subsets of $* N$ which has the finite intersection property and has cardinality $\kappa(\theta)$. By Theorem 2.7.12 of [6] there is an element $\omega$ in the intersection of the collection. That is, $\omega$ is an infinite member of ${ }^{*} N$ and it satisfies $\omega p \epsilon^{*} U$ for every $U$ in $\mathcal{U}$. It follows that $\omega p \in \mu_{\theta}(0)$, which completes the proof.

If the topology $\theta$ is metrizable, then the saturation assumption on $* \pi$ can be removed. In general, however, some saturation assumption seems necessary for this useful result to hold.

Definition 1.7. Let $(E, \theta)$ be a topological vector space in $M$. The nonstandard bull of $(E, \theta)$ is the topological vector space $(\hat{E}, \hat{\theta})$ defined by

(i) $\hat{E}=\operatorname{fin}_{\theta}(* E) / \mu_{\theta}(0)$,

(ii) $\hat{\theta}$ is the quotient topology defined on $\hat{E}$ by $\tilde{\theta}$.

The canonical mapping of fin $\theta$ onto $\hat{E}$ will be denoted by $\pi$. (Thus $\pi(p)=$ $\mu(p)$ for all $p$ in fin $\theta$.) Note that $\pi$ simply identifies those elements of fin $\theta$ which have exactly the same $\widetilde{\theta}$-neighborhood filter in ${ }^{*} E$. Therefore $(\hat{E}, \hat{\theta})$ is a Hausdorff space. If $(E, \theta)$ is a Hausdorff space then the mapping $x \mapsto \mu(x)$ is an (topological vector space) embedding of $(E, \theta)$ into $(\hat{E}, \hat{\theta})$.

Since there is a local base at 0 for $\theta$ of cardinality $\kappa(\theta)$, the uniformity $O(\theta)$ defined on $E$ by $\theta$ can be defined by a set of $\kappa(\theta)$ semimetrics, as is well known. Therefore, it follows from Theorem 3.15.1 of [6] (and the fact that $\tilde{\theta}$ is the topology on ${ }^{*} E$ defined by $\overparen{\mathcal{O}(\theta))}$ that $(\hat{E}, \hat{\theta})$ is a complete space whenever $* \pi$ is $\kappa(\theta)^{+}$-saturated.

In any case, if $(E, \theta)$ is a Hausdorff space, then the closure of (the image of) $E$ in $\hat{E}$ is just $\pi$ (pns $\theta$ ) by Theorem 1.2, and this space is a completion of $(E, \theta)$. Indeed, if $\mathcal{F}$ is any $\theta$-Cauchy filter on $E$ then $\mathcal{F}$ converges in $(\hat{E}, \hat{\theta})$ to $\pi(p)$, where $p \in \mu(\mathcal{F})$.

Now suppose that $\theta$ is the topology defined on $E$ by a norm $\rho$. As noted above, fin $_{\theta}$ is the set of $p$ in ${ }^{*} E$ for which ${ }^{*} \rho(p)$ is finite. Also, $\mu_{\theta}(0)$ is the set of $p$ in ${ }^{*} E$ for which ${ }^{*} \rho(p)$ is infinitesimal. Moreover, the nonstandard hull topology $\hat{\theta}$ 
is defined by the norm $\hat{\rho}$ given on $\hat{E}$ by $\left.\hat{\rho}(x)=s t{ }^{*} \rho(p)\right)$ where $x=\pi(p)$. Thus the nonstandard hulls constructed here for normed spaces are the same as those constructed in [6].

Theorem 1.8. Let $p_{1}, \cdots, p_{n}$ be elements of $\operatorname{fin}_{\theta}\left({ }^{*} E\right)$ such that $\pi\left(p_{1}\right), \cdots$, $\pi\left(p_{n}\right)$ are independent elements of $\hat{E}$ over $K$.

(i) For any $\lambda_{1}, \cdots, \lambda_{n} \in{ }^{*} K, \sum_{j=1}^{n} \lambda_{j} p_{j}=0$ if and only if $\lambda_{1}=\cdots=\lambda_{n}=0$. (That is, $p_{1}, \cdots, p_{n}$ are $*_{\text {-independent over }{ }^{*} K \text {.) }}$

(ii) For any $\lambda_{1}, \cdots, \lambda_{n} \in{ }^{*} K, \sum_{j=1}^{n} \lambda_{j} p_{j}$ is $\theta$-finite if and only if $\lambda_{1}, \cdots, \lambda_{n}$ are all elements of ${ }^{*} K_{0}$. In that case

$$
\pi\left(\sum_{j=1}^{n} \lambda_{j} p_{j}\right)=\sum_{j=1}^{n} s t\left(\lambda_{j}\right) \pi\left(p_{j}\right)
$$

Proof. If $p_{1}, \cdots, p_{n}$ are in fin $_{\theta}$ and $\lambda_{1}, \cdots, \lambda_{n}$ are in ${ }^{*} K_{0}$, then $\sum_{j=1}^{n} \lambda_{j} p_{j} \epsilon$ fin $_{\theta}$ by Theorem 1.2. By Theorem 1.3, for each $j=1, \cdots, n,\left[\lambda_{j}-\text { st }\left(\lambda_{j}\right)\right]_{p_{j}}$ is in $\mu_{\theta}(0)$. Thus

$$
\pi\left(\sum_{j=1}^{n} \lambda_{j} p_{j}\right)-\sum_{j=1}^{n} \mathrm{st}\left(\lambda_{j}\right) \pi\left(p_{j}\right)=\pi\left(\sum_{j=1}^{n}\left[\lambda_{j}-\mathrm{st}\left(\lambda_{j}\right)\right] p_{j}\right)=0
$$

and one part of (ii) has been proved.

Now suppose $\pi\left(p_{1}\right), \cdots, \pi\left(p_{n}\right)$ are independent in $\hat{E}$ and $\lambda_{1}, \ldots, \lambda_{n}$ are in ${ }^{*} K$. Assume that $\sum_{j=1}^{n} \lambda_{j} p_{j}=0$. If not all $\lambda_{j}$ are 0 , we may assume $\lambda_{1} \neq 0$ and $\left|\lambda_{1}\right| \geq$ $\left|\lambda_{j}\right|$ for $j=1, \cdots, n$. Then $\sum_{j=1}^{n}\left(\lambda_{j} /\left|\lambda_{1}\right|\right) p_{j}=0$. Applying the previous argument and using the independence of $\pi\left(p_{1}\right), \cdots, \pi\left(p_{n}\right)$ leads to the conclusion that st $\left(\lambda_{j} /\left|\lambda_{1}\right|\right)=0$ for $j=1, \cdots, n$. But this is a contradiction for $j=1$. Therefore $\lambda_{1}$ $=\cdots=\lambda_{n}=0$, and (i) has been proved.

To finish the proof of (ii), suppose $\pi\left(p_{1}\right), \cdots, \pi\left(p_{n}\right)$ are independent and $\lambda_{1}$, $\cdots, \lambda_{n}$ are in ${ }^{*} K$, and that

$$
\sum_{j=1}^{n} \lambda_{j} p_{j} \in \operatorname{fin}_{\theta}
$$

We may assume that $\left|\lambda_{1}\right| \geq \cdots \geq\left|\lambda_{n}\right|$. If $\lambda_{1}$ is not in ${ }^{*} K_{0}$, then by Theorem 1.3

$$
\sum_{j=1}^{n} \frac{\lambda_{j}}{\left|\lambda_{1}\right|} p_{j} \in \mu_{\theta}(0)
$$

But this implies that st $\left(\lambda_{1} /\left|\lambda_{1}\right|\right)=0$, as above, which is a contradiction. Therefore, $\lambda_{1}, \cdots, \lambda_{n}$ are all in ${ }^{*} K_{0}$, and the proof is complete.

2. $\theta$-bounded elements of $* E$. Let $(E, \theta)$ be a topological vector space which is an element of $M$. 
Theorem 2.1. A subset $B$ of $E$ is $\theta$-bounded if and only if ${ }^{*} B \subset$ fin $_{\theta}\left({ }^{*} E\right)$.

Proof. Assume $B$ is a $\theta$-bounded subset of $E$. Given any $\theta$-neighborhood $U$ of 0 , there exists $n \in N$ such that $B \subset n U$, and therefore ${ }^{*} B \subset n^{*} U$. Hence every element of ${ }^{*} B$ is $\theta$-finite.

Conversely, assume that $B$ is a subset of $E$ and every element of ${ }^{*} B$ is $\theta$ finite. If $U$ is any $\theta$-neighborhood of 0 , it follows from Theorem 1.3 that the internal set $\left\{\left.\omega\right|^{*} B \subset \omega^{*} U\right\}$ contains every infinite member of ${ }^{*} N$. Therefore, it contains some standard integer $n$, which thus satisfies $B \subset n U$. It follows that $B$ is $\theta$-bounded.

Definition 2.2. An element $p$ of ${ }^{*} E$ is $\theta$-bounded if there exists a $\theta$-bounded subset $B$ of $E$ which satisfies $p \epsilon^{*} B$.

The set of $\theta$-bounded elements of ${ }^{*} E$ will be denoted by bd ${ }_{\theta}\left({ }^{*} E\right)$, or simply by bd $\theta$.

Theorem 2.3. (i) bd ${ }^{(* E)}$ is closed under addition and under multiplication by elements of ${ }^{*} K_{0}$.

(ii) $*[E] \subset$ bd $_{\theta}(* E) \subset \operatorname{fin}_{\theta}(* E)$.

Proof. (i) Let $p$ and $q$ be $\theta$-bounded elements of ${ }^{*} E$. Since the absolutely convex hull of the union of two $\theta$-bounded sets is again $\theta$-bounded, it follows that there is an absolutely convex, $\theta$-bounded subset $B$ of $E$ such that $p, q \in{ }^{*} B$. Then $p+$ $q \in *(2 B)$ so that $p+q$ is $\theta$-bounded. If $\lambda$ is in ${ }^{*} K_{0}$, then there is an integer $n$ in $N$ with $|\lambda| \leq n$. Since $B$ is circled, $\lambda p \epsilon^{*}(n B) \subset$ bd $\theta$.

(ii) This follows immediately from the definition and Theorem 2.1.

Theorem 2.4. A subset $B$ of $E$ is $\theta$-totally bounded if and only if ${ }^{*} B C$ pns ${ }_{\theta}(* E)$.

Proof. Let $B$ be a $\theta$-totally bounded subset of $E$ and let $p \epsilon^{*} B$. Given any $\theta$-neighborhood $U$ of 0 , there exist $x_{1}, \cdots, x_{n}$ in $E$ which satisfy

$$
B \subset \bigcup\left\{x_{i}+U \mid i=1, \cdots, n\right\} .
$$

Therefore $p \in \epsilon^{*} x_{i}+{ }^{*} U$ for some $i=1, \cdots, n$. This shows that $p$ is in the $\tilde{\theta}$-closure of $*[E]$, and therefore $p$ is $\theta$-pre-nearstandard by Theorem 1.2. Thus ${ }^{*} B \subset$ pns $\theta$.

Conversely, assume ${ }^{*} B C$ pns $\theta$. If $B$ is not $\theta$-totally bounded, then there exists a $\theta$-neighborhood $U$ of 0 such that for every $x_{1}, \cdots, x_{n}$ in $E$

$$
B \sim \bigcup\left\{x_{i}+U \mid i=1, \cdots, n\right\} \neq \varnothing
$$

Since $* M$ is an enlargement, it follows that there is an element $p$ of ${ }^{*} E$ which satisfies $p \epsilon^{*} B$ and $p \notin^{*} x+{ }^{*} U$ for every $x$ in $E$. By Theorem 1.2 again, this shows that $p$ is not $\theta$-pre-nearstandard. This contradiction proves that $B$ is $\theta$-totally bounded. 
Theorem 2.5. The following conditions are equivalent:

(i) every $\theta$-bounded subset of $E$ is relatively $\theta$-compact,

(ii) $\mathrm{bd}_{\theta} \subset \mathrm{ns} \theta$.

Proof. The vector topology $\theta$ on $E$ is regular, so that Theorem 3.6.1 of [6] applies to $(E, \theta)$. Therefore a subset $B$ of $E$ is relatively $\theta$-compact if and only if ${ }^{*} B \subset \mathrm{ns} \theta$. The equivalence of (i) and (ii) is an immediate consequence of that fact.

The following result is an immediate consequence of Theorem 2.4.

Theorem 2.6. The following conditions are equivalent:

(i) every $\theta$-bounded subset of $E$ is $\theta$-totally bounded,

(ii) $b_{\theta} \subset$ pns $\theta$.

Theorem 2.7. The following conditions are equivalent:

(i) $\theta$ is defined on $E$ by a seminorm,

(ii) $b_{\theta}=$ fin $_{\theta}$,

(iii) $\mu_{\theta}(0) \subset$ bd $\theta$.

Proof. Recall that (i) is equivalent to the statement that there exists a $\theta$-bounded $\theta$-neighborhood of 0 .

(i) implies (ii). Let $B$ be a $\theta$-bounded, $\theta$-neighborhood of 0 . If $p$ is $\theta$-finite, then $p \in n^{*} B$ for some $n$ in $N$. It follows that $p$ is $\theta$-bounded and hence fin ${ }_{\theta} C$ bd $\theta$. The equality (ii) is a consequence of Theorem 2.3.

(ii) implies (iii). This follows immediately from the fact that $\mu_{\theta}(0) \subset$ fin $\theta$.

(iii) implies (i). Let $\mathcal{U}$ be the filter of $\theta$-neighborhoods of 0 and let $B$ be the collection of all $\theta$-bounded subsets of $E$. If there is no $\theta$-bounded set in $\mathcal{U}$, then $E$ is not a union of finitely many elements of $\mathfrak{B}$. Therefore $\mathcal{F}=\{E \sim B \mid B \in \mathfrak{B}\}$ has the finite intersection property. Moreover, if $A$ is in $\mathcal{F}$, then $* A \cap \mu_{\theta}(0) \neq \emptyset$. Since the monad of $\mathcal{F}$ is equal to ${ }^{*} E \sim \mathrm{bd}_{\theta}$, it follows from Theorem 2.7.1 of [6] that $\mu_{\theta}(0) \cap\left({ }^{*} E \sim \operatorname{bd}_{\theta}\right) \neq \emptyset$. Therefore (iii) implies that there must be a $\theta$-bounded set in $\mathcal{U}$, and hence that $\theta$ is defined on $E$ by a seminorm.

3. Finite elements of ${ }^{*} E$ relative to the $\theta$-uniformity on $E$. Let $(E, \theta)$ be a topological vector space in $\mathbb{M}$ and let $\mathcal{U}$ be the filter of $\theta$-neighborhoods of 0 . For each $U$ in $\mathcal{U}$ define $V(U)=\{(x, y) \mid x-y \in U\}$ (a subset of $E \times E$ ). Let $\vartheta(\theta)$ be the filter on $E \times E$ generated by the filter base $\{V(U) \mid U \in \mathcal{U}\}$. Then $\mathcal{O}(\theta)$ is the unique translation invariant uniformity on $E$ which determines the topology $\theta$. In [3] the concept (for elements of ${ }^{*} E$ ) of finiteness with respect to a uniformity is introduced. In this section we compare $O(\theta)$-finiteness with the concept of $\theta$-finiteness defined in $\$ 1$.

Definition 3.1. [3] An element $p$ of ${ }^{*} E$ is $\mathcal{O}(\theta)$-finite if for each $V$ in $\mathcal{O}(\theta)$ there exist $n$ in $N$ and $x$ in $E$ such that $\left(p,{ }^{*} x\right) \epsilon^{*}\left(V^{n}\right)$. 
The set of $\vartheta(\theta)$-finite elements of ${ }^{*} E$ will be denoted by fin $\vartheta(\theta)$.

Lemma 3.2. If $p \epsilon^{*} E$, then $p$ is $\mathcal{O}(\theta)$-finite if and only if for each $\theta$-neigbborbood $U$ of 0 there exists $n$ in $N$ such that $p \in *(U+\cdots+U)$ ( $n$ times).

Proof. Since the collection $\{V(U) \mid U \in \mathcal{U}\}$ is a filter base for $\mathcal{O}(\theta)$, it follows that $p$ is $\mathcal{O}(\theta)$-finite if and only if for each $\theta$-neighborhood $U$ of 0 there exist $m$ in $N$ and $x$ in $E$ such that $\left(p,{ }^{*} x\right) \in *\left(V(U)^{m}\right)$. This is, in turn, equivalent to $p \epsilon$ $*_{x}+*(U+\cdots+U)(m$ times $)=*(x+(U+\cdots+U))(m$ times $)$. But for each $x$ in $E$ and each neighborhood $U$ of 0 , there exists $k$ in $N$ such that $x \in k U \subset U+\cdots+U$ ( $k$ times). Thus the result follows.

If $U$ is any subset of $E$ which has 0 as an element, then for each $n$ in $N$, $n U \subset U+\cdots+U$ ( $n$ times); if, in addition, $U$ is convex, then $n U=U+\cdots+U(n$ times). The following theorem is an easy consequence of this remark and Lemma 3.2.

Theorem 3.3. (i) if $(E, \theta)$ is a topological vector space in $\mathbb{M}$, then fin $\left.\theta_{(} \theta\right)$ is a K-subspace of ${ }^{*} E$ and $\operatorname{fin}_{\theta} \subset$ fin $\mathrm{O}(\theta)^{\text {. }}$

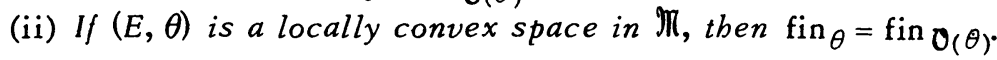

The following example shows that it is possible for fin $\theta$ to be a proper $K$-subspace of fin $\theta_{(\theta)}$. By Theorem 1.2 this implies $\tilde{\theta}$ need not be a vector topology on fin $\left.\theta_{(} \theta\right)^{\circ}$

Example 3.4. Let $E$ be the vector space of all real valued sequences $x=$ $(x(1), x(2), \ldots)$ such that $\{n \mid x(n) \neq 0\}$ is finite. Define a function $d$ on $E$ by

$$
d(x)=\sum_{n=1}^{\infty}|x(n)|^{1 / n} .
$$

If $a$ and $b$ are in $R^{+}$and $n$ is in $N$; then $(a+b)^{1 / n} \leq a^{1 / n}+b^{1 / n}$. Hence

(i) if $x, y \in E$, then $d(x+y) \leq d(x)+d(y)$.

Now suppose $x$ is in $E$ and $|\lambda| \leq 1$. For some $k$ in $N$, if $n>k$ then $x(n)=0$. Therefore

(ii) $d(\lambda x)=\sum_{n=1}^{k}|\lambda|^{1 / n} \cdot|x(n)|^{1 / n} \leq|\lambda|^{1 / k} \cdot d(x)$.

For each $j$ in $N$ let

$$
U_{j}=\{x \mid x \in E \text { and } d(x) \leq 1 / j\}
$$

and let $\mathcal{U}$ be the filter on $E$ generated by $\left\{U_{j} \mid j \in N\right\}$. By (ii), each $U_{j}$ is absorbing and circled. By (i), $U_{j}$ contains $U_{2 j}+U_{2 j}$ for each $j$ in $N$. Thus there is a unique vector topology $\theta$ on $E$ such that $\mathcal{U}$ is the $\theta$-neighborhood filter of 0 .

Pick an infinite integer $\omega$ in ${ }^{*} N$ which is divisible by every standard integer $n$ in $N$. (For example, let $\omega=\left(\omega^{\prime}\right)$ ! where $\omega^{\prime}$ is an infinite integer.) Pick $\tau$ in ${ }^{*} N$ such that $(1 / \omega)^{1 / \tau}>1 / 2$. Define an element $p$ of ${ }^{*} E$ (as a function from ${ }^{*} N$ into ${ }^{*} R$ ) by 


$$
p(n)= \begin{cases}(1 / \omega)^{n} & \text { if } \tau \leq n<\tau+\omega, \\ 0 & \text { otherwise. }\end{cases}
$$

If $p$ is $\theta$-finite, then, by Theorem $1.3,(1 / \omega) p \in \mu_{\theta}(0)$ and therefore ${ }^{*} d((1 / \omega) p)$ $={ }_{1}$. But

$$
{ }^{*} d\left(\frac{1}{\omega}\right) p=\sum_{n=\tau}^{\tau+\omega-1}\left(\frac{1}{\omega}\right)^{1 / n} \cdot\left(\frac{1}{\omega}\right) \geq\left(\frac{1}{\omega}\right)^{1 / \tau} \cdot\left(\frac{\omega}{\omega}\right)>\frac{1}{2},
$$

which is a contradiction. Hence $p$ is not $\theta$-finite.

It remains to show that $p$ is $\vartheta(\theta)$-finite. Let $j$ be in $N$. Then for some $\omega^{\prime}$ in $* N, j \omega^{\prime}=\omega$. For each $i, 1 \leq i \leq j$, define $p_{i}$ in $E$ by

$$
p_{i}(n)= \begin{cases}p(n) & \text { if } \tau+(i-1) \omega^{\prime} \leq n<\tau+i \omega^{\prime}, \\ 0 & \text { otherwise. }\end{cases}
$$

Clearly, $p=p_{1}+\cdots+p_{j}$. But for each $i, * d\left(p_{i}\right) \leq \omega^{\prime} / \omega=1 / j$ and therefore $p_{i} \in{ }^{*} U_{j}$. Thus $p$ $\epsilon *\left(U_{j}+\cdots+U_{j}\right)$ ( $j$ times) for each $j$ in $N$. It follows by Lemma 3.2 that $p$ is $\mathcal{O}(\theta)$-finite.

Remark. Let $(E, \theta)$ be a topological vector space in $\mathbb{M}$ and let $\mathcal{S}$ be any set of semimetrics on $E$ which defines the uniformity $\mathcal{O}(\theta)$ on $E$. In [6] Luxemburg defines a set $F$ of "finite" elements of ${ }^{*} E$ by

$$
F=\left\{p \mid p \epsilon^{*} E \text { and }{ }^{*} d(0, p) \text { is finite for all } d \in \mathfrak{S}\right\}
$$

and sets $E_{0}=\{\mu(p) \mid p \in F\}$. He uses $\mathcal{S}$ to construct a uniformity $\mathcal{O}_{0}$ on $E_{0}$, and refers to $\left(E_{0}, \mathcal{O}_{0}\right)$ as a nonstandard hull of the uniform space $(E, \mathcal{O}(\theta))$.

Let $\theta_{0}$ be the topology defined on $E_{0}$ by $\mathcal{O}_{0}$. As was discussed in [3], the set $F$ always contains fin $_{\theta(\theta)}$ and $\theta_{0}$ is the same as the quotient topology obtained on $E_{0}$ from the restriction of $\tilde{\theta}$ to $F$. Therefore if $(E, \theta)$ is the space in Example 3.4 , then $\left(E_{0}, \theta_{0}\right)$ is never a topological vector space.

4. Invariance of the nonstandard hulls. Let $(E, \theta)$ be a Hausdorff topological vector space in $M$. It was noted in $\oint_{1}$ that $(E, \theta)$ can be embedded in the nonstandard hull $(\hat{E}, \hat{\theta})$ and, in fact, the image of pns ${ }_{\theta}$ in $\hat{E}$ is a completion of $(E, \theta)$. On the other hand, if $* M$ is $\kappa$-saturated for $\kappa>\kappa(\theta)$ then $(\hat{E}, \hat{\theta})$ is complete. In this section we investigate the question of when $(\hat{E}, \hat{\theta})$ itself is a completion of $(E, \theta)$. Since $p \in$ pns $_{\theta}$ implies $\mu(p) \subset$ pns $_{\theta}$, it is clear that $(\hat{E}, \hat{\theta})$ is a completion of $(E, \theta)$ if and only if pns $\theta=f_{\theta}$. However, this is in turn equivalent to a standard condition on $(E, \theta)$ and so is independent of the particular enlargement $* M$ being considered.

Theorem 4.1. Let $\mathcal{U}$ be the $\theta$-neigbborbood filter of 0 in E. Then $\mathrm{pns}_{\theta}=$ fin $_{\theta}$ if and only if every ultrafilter $\mathcal{F}$ on $E$ which satisfies the condition 
is a Caucby filter.

Proof. Assume pns ${ }_{\theta}=\mathrm{fin}_{\theta}$ and $\mathcal{F}$ is an ultrafilter on $E$ satisfying $(*)$. By Theorem 1.4, $\mu(\mathfrak{F}) \subset$ fin $_{\theta}=$ pns $_{\theta}$. Thus if $p \in \mu(\mathfrak{F})$, then by Theorem 3.12.1 of [6] there exists a Cauchy filter $\mathcal{G}$ on $E$ such that $\mu(p)=\mu(\mathcal{G})$. But $\mathcal{F}$ is an ultrafilter, so that $\mu(\mathfrak{F}) \subset \mu\left(\mathcal{G}_{)}\right.$and $\mathfrak{F}$ is also a Cauchy filter.

Assume that every ultrafilter satisfying condition $(*)$ is a Cauchy filter. Now

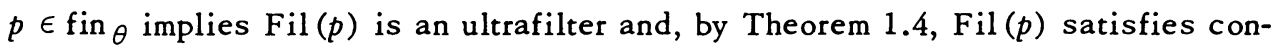
dition $(*)$. Thus Fil $(p)$ is a Cauchy filter. By Theorem 3.12.3 of [6], $p$ is in pns $\theta$. Thus fin ${ }_{\theta} \subset$ pns $_{\theta}$ and equality follows from Theorem 1.2.

Note that if $(E, \theta)$ is a topological vector space satisfying the condition of Theorem 4.1, then all the nonstandard hulls of $(E, \theta)$ are isomorphic. In Theorem 4.3 it is shown that if the condition of Theorem 4.1 is not satisfied then the nonstandard hulls are not isomorphic; indeed, there are nonstandard hulls of arbitrarily large cardinality in that case. The following result, from which Theorem 4.3 follows, is of interest in its own right.

Lemma 4.2 Assume that $* M$ is $\kappa$-saturated. If $p \in{ }^{*} E$ is not in pns $\theta$ and $A$ is any subset of ${ }^{*} E$ which satisfies

$$
\mu(\mathrm{Fil}(p)) \subset \bigcup\{\mu(q) \mid q \in A\}
$$

then $\operatorname{card}(A) \geq \kappa$.

Proof. Let $p \in{ }^{*} E \sim$ pns $_{\theta}$ and $\mathcal{F}=\mathrm{Fil}(p)$. Assume that $A$ is an arbitrary subset of ${ }^{*} E$ which satisfies $\mu(\mathcal{F}) \subset \bigcup\{\mu(q) \mid q \in A\}$. Suppose also that $A$ has cardinality $<\kappa$.

First it will be shown that there is a *-finite subset $B$ of ${ }^{*} E$ which satisfies $A \subset B$. For each $q \in A$ let $S(q)$ be the internal set

$$
S(q)=\left\{B \mid B \text { is a }{ }^{*} \text {-finite subset of }{ }^{*} E \text { and } q \in B\right\} \text {. }
$$

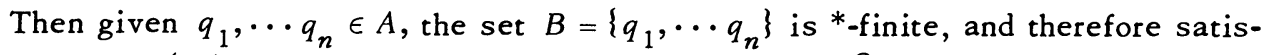
fies $B \in S\left(q_{1}\right) \cap \ldots \cap S\left(q_{n}\right)$. Therefore, the collection $\mathbb{Q}=\{S(q) \mid q \in A\}$ has the finite intersection property. Since $\mathbb{Q}$ has cardinality less than $\kappa$, it follows from Theorem 2.7.12 of [6] that some set $B$ is in the intersection of $\mathbb{Q}$. That is, $B$ is a $*_{\text {-finite subset of }}^{*} E$ and $q \in B$ for every $q \in A$.

Next it will be shown that $\mathcal{F}$ is a Cauchy filter in $(E, \theta)$. Let $U$ be a $\theta$-neighborhood of 0 . Pick a circled $\theta$-neighborhood $V$ of 0 such that $V+V \subset U$. Now if $W \in * \mathfrak{F}$ satisfies $W \subset \mu(\mathfrak{F})$, then $W \subset \bigcup\left\{q+{ }^{*} V \mid q \in B\right\}$. Since $\mathcal{F}$ is an ultrafilter and $B$ is *-finite, it follows that $q_{0}+* V \in * \mathcal{F}$ for some $q_{0}$ in $B$. But then for some $x_{0} \subset E$ it follows that $F=x_{0}+V \in \mathcal{F}$. Now $F-F=\left(x_{0}+V\right)-\left(x_{0}+V\right)=$ $V-V \subset U$. Since $U$ is arbitrary this shows that $\mathcal{F}$ is a Cauchy filter. 
Hence $\mu(\mathcal{F}) \subset \mu(p)$ and $p \in \operatorname{pns}_{\theta}[6$, Theorem 3.12.3], which is a contradiction. Thus $\operatorname{card}(A) \geq \kappa$.

Theorem 4.3. Let $(E, \theta)$ be a Hausdorff topological vector space and assume $* \mathbb{N}$ is $\kappa$-saturated where $\kappa>\kappa(\theta)$. If pns ${ }_{\theta} \neq$ fin $_{\theta}$, then the nonstandard bull $(\hat{E}, \hat{\theta})$ is a complete topological vector space of cardinality greater than or equal to $\kappa$.

Proof. It was noted in $\$ 1$ that $(\hat{E}, \hat{\theta})$ is a complete space when $* \pi$ is $\kappa(\theta)+$

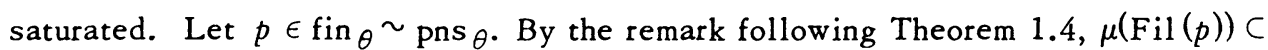
fin $_{\theta}$ and, by Lemma 4.2 , the image of $\mu(\mathrm{Fil}(p))$ in $\hat{E}$ has cardinality greater than or equal to $\kappa$. In particular, $\hat{E}$ has cardinality at least $\kappa$.

Remark. Let $(E, \theta)$ be a Hausdorff topological vector space. Theorems 4.1 and 4.3 show that either the nonstandard hulls of $(E, \theta)$ are all isomorphic to a completion of $(E, \theta)$ (and hence to each other) or there are nonstandard hulls of $(E, \theta)$ of arbitrarily large cardinality. In the latter case every nonstandard hull contains a completion of $(E, \theta)$ as a proper subspace.

In the former case we say that the nonstandard bulls of $(E, \theta)$ are invariant.

Theorem 4.4. The nonstandard hullls of a normed space $(E, \rho)$ are invariant if and only if $E$ is finite dimensional.

Proof. Clearly, if $E$ is finite dimensional, then the nonstandard hulls of $(E, \rho)$ are invariant.

Suppose $E$ is infinite dimensional, so that the closed unit ball of $(E, \rho)$ is bounded but not totally bounded. It follows from Theorems 2.1 and 2.4 that pns $\theta \neq$ fin $\theta$. The result follows from Theorems 4.1 and 4.3 .

Remark. A more direct proof of Theorem 4.4 is possible, as follows. If $E$ is infinite dimensional, then the closed unit ball of $(E, \rho)$ is not totally bounded. Thus there exist $\delta>0$ in $R$ and a sequence $\left\{x_{n} \mid n \in N\right\}$ in $E$ such that $\rho\left(x_{n}\right) \leq 1$ for $n$ in $N$ and $\rho\left(x_{n}-x_{m}\right)>\delta$ for distinct $n, m$ in $N$. Then for each $\omega$ in ${ }^{*} N$,

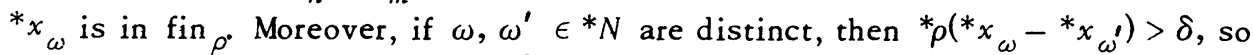
that $\left.\left.\pi^{*} x_{\omega}\right) \neq \pi^{*} x_{\omega}^{\prime}\right)$. Therefore $\hat{E}$ has cardinality at least as great as the cardinality of $* N$. Since the enlargement $* \pi$ can be chosen so that $* N$ has arbitrarily large cardinality, it follows that the nonstandard hulls of $(E, \rho)$ are not invariant.

However, the following example shows that there do exist infinite dimensional, metrizable, locally convex spaces with invariant nonstandard hulls.

Example 4.5. Let $D$ be a nonempty open subset of the complex plane, and let $E$ be the space of all functions which are analytic on $D$. Let $\theta$ be the topology of uniform convergence on compact subsets. Then $(E, \theta)$ is a complete metrizable locally convex space and $E$ is infinite dimensional.

Using results of Robinson [9, pp. 155-158] we show that fin $\theta=$ ns $\theta$. Let $B=$ $\bigcup\{* C \mid C \subset D$ and $C$ is compact\}. Now let $p \in$ fin $\theta$. Then for each compact subset $C$ of $D, p$ is bounded by a standard real number on ${ }^{*} C$. Thus if $w \in B$ then $p(w)$ 
is finite. Since $*[D] \subset B$, we may define $f$ on $D$ by $f(z)=$ st $\left.\left(p^{*} z\right)\right)$. Robinson shows in Theorem 6.2.3 of [9] that $f$ is analytic on $D$. Moreover, if $C$ is a compact subset of $D$, then $p(w)={ }_{1} * f(w)$ for all $w$ in ${ }^{*} C$. (This requires an argument in addition to that necessary to show that $f$ is analytic. In fact $p$ is $S$-continuous, in Robinson's terminology, by Theorem 6.2.2 of [9].) But this means that $p$ is $\theta$ nearstandard to $f$; hence $\operatorname{fin}_{\theta}=\mathrm{ns} \theta$.

In $\$ 5$ it is shown that if $(E, \theta)$ is a Hausdorff locally convex space with dual space $E^{\prime}$ and if $\theta$ is the weak topology $\sigma\left(E, E^{\prime}\right)$, then pns $\theta=f_{\theta}$. (Theorem 5.13.)

The following theorem shows that every nonstandard hull can be obtained as the nonstandard hull of a complete space.

Theorem 4.6. Let $(G, \Delta)$ be a Hausdorff topological vector space and let $(E, \theta)$ be a dense subspace of $(G, \Delta)$. Then

(i) $\operatorname{pns}_{\theta}(* E)=\operatorname{pns}_{\Delta}(* G) \cap * E$;

(ii) $\operatorname{fin}_{\theta}\left({ }^{*} E\right)=\operatorname{fin}_{\Delta}(* G) \cap{ }^{*} E$ and the quotient mapping $\pi:$ fin $_{\Delta}(* G) \rightarrow \hat{G}$ restricted to $\operatorname{fin}_{\theta}\left({ }^{*} E\right.$ ) induces an isomorphism (as topological vector spaces) of $(\hat{E}, \hat{\theta})$ onto $(\hat{G}, \hat{\Delta})$.

Proof. (i) Clearly pns ${ }_{\theta}\left({ }^{*} E\right) \subset$ pns ${ }_{\Delta}(* G) \cap{ }^{*} E$. Suppose $p \in$ pns $_{\Delta}(* G) \cap * E$ and $U$ is a $\theta$-neighborhood of 0 in $E$. There is a $\Delta$-neighborhood $W$ of 0 in $G$ such that $U=W \cap E$. Pick a $\Delta$-neighborhood $V$ of 0 in $G$ such that $V+V \subset W$. There exists $x$ in $G$ such that $p \in{ }^{*} x+{ }^{*} V$. Since $E$ is dense in $G$ there exists $y$ in $E$ such that $x \in y+V$. Thus $p-{ }^{*} y \epsilon^{*} V+{ }^{*} V \subset{ }^{*} W$, and $p-{ }^{*} y \in \epsilon^{*} U$. Hence $p$ is in the $\tilde{\theta}$-closure of $*[E]$, that is $p \in$ pns $_{\theta}(* E)$. Thus $\operatorname{pns}_{\Delta}(* G) \cap * E C$ pns ${ }_{\theta}(* E)$.

(ii) The equality fin ${ }_{\theta}(* E)=$ fin $_{\Delta}(* G) \cap{ }^{*} E$ follows immediately from Definition 1.1. It follows that $\pi$ induces an isomorphism of $(\hat{E}, \hat{\theta})$ into $(\hat{G}, \hat{\Delta})$. In order to show that this isomorphism is onto $\hat{G}$, it is sufficient to show that, for each $p$ in ${ }^{*} G,\left(p+\mu_{\Delta}(0)\right) \cap{ }^{*} E \neq \emptyset$. But let $p \in{ }^{*} G$ and let $W$ be a $* \Delta$-neighborhood of 0 such that $W \subset \mu_{\Delta}(0)$. Then since $E$ is dense in $G$ there exists $q$ in ${ }^{*} E$ such that $q-p \in W$. So $q \in p+W \subset p+\mu_{\Delta}(0)$ and the proof is complete.

In particular, if $(E, \theta)$ is a Hausdorff topological vector space and $(G, \Delta)$ is its completion, then $(\hat{E}, \hat{\theta})$ and $(\hat{G}, \hat{\Delta})$ are isomorphic. In addition by Theorem 4.6 the nonstandard hulls of $(E, \theta)$ are invariant if and only if the nonstandard hulls of $(G, \Delta)$ are invariant. This is in turn equivalent to the condition that each nonstandard hull of $(G, \Delta)$ be isomorphic to $(G, \Delta)$.

It is an open question which complete topological vector spaces have invariant nonstandard hulls. Note that by Theorem 2.5 a necessary condition for the nonstandard hulls of $(E, \theta)$ to be invariant is that every $\theta$-bounded set in $E$ be relatively $\theta$-compact. 
5. Pairings. Let $E$ be a vector space over $K$ which is an element of $M$.

Definition 5.1. Let $A$ be a subset of ${ }^{*} E$ (internal or external). The byperconvex bull of $A$ is the set of all elements of ${ }^{*} E$ of the form $\sum_{k=1}^{\omega} \lambda_{k} p_{k}$ where $\omega \epsilon^{*} N$, $\left\{p_{1}, p_{2}, \cdots p_{\omega}\right\}$ is an internal sequence of elements of $A$ and $\left\{\lambda_{1}, \lambda_{2}, \cdots, \lambda_{\omega}\right\}$ is an internal sequence of elements of ${ }^{*} R^{+}$which satisfies $\sum_{k=1}^{\omega} \lambda_{k}=1$. A is byperconvex if it is equal to its hyperconvex hull.

If $\mathcal{C}$ is the set of all convex subsets of $E$ and if $A$ is an internal subset of ${ }^{*} E$, then $A$ is hyperconvex if and only if $A \in * \mathcal{C}$. In particular, if $C \subset E$ is convex, then ${ }^{*} C$ is hyperconvex. The hyperconvex hull of any internal set is again internal. In general, the hyperconvex hull of $A$ is the smallest hyperconvex set containing $A$. If $B \subset E$ and $C$ is the convex hull of $B$, then ${ }^{*} C$ is the hyperconvex hull of ${ }^{*} B$. Note also that the intersection of a family of hyperconvex sets is again a hyperconvex set. In particular, if $\theta$ is a locally convex vector topology on $E$, then $\mu_{\theta}(0)$ is a hyperconvex set.

Definition 5.2. Let $A$ be a subset of ${ }^{*} E$ (internai or external).

(i) The bypercircled bull of $A$ is the set of all elements of ${ }^{*} E$ of the form $\lambda p$, where $p \in A, \lambda \in{ }^{*} K$, and $|\lambda| \leq 1$. $A$ is bypercircled if it is equal to its hypercircled hull.

(ii) The absolutely byperconvex bull of $A$ is the set of all elements of ${ }^{*} E$ of the form $\sum_{k=1}^{\omega} \lambda_{k} p_{k}$ where $\omega \in \epsilon^{*} N,\left\{p_{1}, p_{2}, \cdots p_{\omega}\right\}$ is an internal sequence of elements of $A$ and $\left\{\lambda_{1}, \lambda_{2}, \cdots \lambda_{\omega}\right\}$ is an internal sequence of elements of ${ }^{*} K$ which satisfies $\sum_{k=1}^{\omega}\left|\lambda_{k}\right| \leq 1$. A is absolutely byperconvex if it is equal to its absolutely hyperconvex hull.

Analogous statements to those made above for the hyperconvex sets can be made for the hypercircled sets and for the absolutely hyperconvex sets. Also note that a subset $A$ of ${ }^{*} E$ is absolutely hyperconvex if and only if it is hyperconvex and hypercircled.

Theorem 5.3. If $\theta$ is a vector topology on $E$, then $(E, \theta)$ is a locally convex space if and only if $\mu_{\theta}(0)$ is byperconvex.

Proof. As noted above if $(E, \theta)$ is locally convex, then $\mu_{\theta}(0)$ is the intersec tion of a family of hyperconvex sets and is therefore hyperconvex itself.

Conversely, suppose $\mu_{\theta}(0)$ is hyperconvex. Let $\mathcal{U}$ be the $\theta$-neighborhood filter at 0 , and let $U \in \mathcal{U}$. Choose $V \in * \mathcal{U}$ which satisfies $V \subset \mu_{\theta}(0)$. Then the hyperconvex hull of $V$ is also contained in $\mu_{\theta}(0)$, and thus also in ${ }^{*} U$. Passing this condition on $U$ back to $M$, it follows that there exists an element $W$ of $U$ whose convex hull is contained in $U$. Thus $(E, \theta)$ is locally convex.

Now let $F$ be another vector space over $K$ (also in $\mathbb{M}$ ) and let $(\ldots, \ldots)$ be a pairing between $E$ and $F$. The image in $* \Re$ of the function $(x, y) \mapsto\langle x, y\rangle$ from $E \times F$ to $K$ is an internal function from ${ }^{*} E \times{ }^{*} F$ to ${ }^{*} K$, which we also denote by $\langle\ldots, \cdots\rangle$. 
Definition 5.4. Let $A$ be a subset of ${ }^{*} E$ (internal or external).

(i) $A^{0}=\left\{q \mid q \epsilon^{*} F\right.$ and $|\langle p, q\rangle| \leq 1$ for all $\left.p \in A\right\}$,

(ii) $A^{i}=\left\{q \mid q \in{ }^{*} F\right.$ and $\langle p, q\rangle \in{ }^{*} K_{1}$ for all $\left.p \in A\right\}$,

(iii) $A^{f}=\left\{q \mid q \in \epsilon^{*} F\right.$ and $\langle p, q\rangle \in{ }^{*} K_{0}$ for all $\left.p \in A\right\}$.

Following the usual convention, we denote $\left(A^{i}\right)^{i}$ by $A^{i i},\left(A^{f}\right)^{0}$ by $A^{f 0}$, etc.

Lemma 5.5. Let $A$ and $B$ be subsets of ${ }^{*} E$.

(i) $A^{i} \subset A^{0} \subset A^{f}$;

(ii) $A^{i}, A^{0}$, and $A^{f}$ are absolutely byperconvex;

(iii) $A^{i}$ and $A^{f}$ are closed under addition and under multiplication by elements of $* K_{0}$;

(iv) if $A \subset B$, then $B^{i} \subset A^{i}, B^{0} \subset A^{0}$, and $B^{f} \subset A^{f}$;

(v) if $A$ is closed under multiplication by elements of $N$, then $A^{i}=A^{0}$;

(vi) $A \subset A^{00} \subset A^{i i} \subset A^{f f}=A^{i f}$;

(vii) $A^{i i i}=A^{i}, A^{000}=A^{0}$ and $A^{f f f}=A^{f}$;

(viii) if $A=* S$ for some $S \subset E$, then $A^{0}=*\left(S^{0}\right)$.

Proof. Parts (i), (iii), (iv) and (viii) are obvious. Part (ii) follows from the fact that ${ }^{*} K_{0},{ }^{*} K_{1}$ and $\left\{\lambda \mid \lambda \epsilon{ }^{*} K\right.$ and $\left.|\lambda| \leq 1\right\}$ are all absolutely hyperconvex. Part (vii) is an immediate consequence of (iv) and (vi).

Part (v). Suppose $A$ is closed under multiplication by elements of $N$. If $q \epsilon$ $A^{0}$ and $p \in A$, then $|\langle n p, q\rangle| \leq 1$ for every $n$ in $N$, and therefore $\langle p, q\rangle \in{ }^{*} K_{1}$. This shows that $A^{0} \subset A^{i}$. The equality $A^{0}=A^{i}$ now follows from (i).

Part (vi). Obviously $A \subset A^{00}$. Since $A^{i} \subset A^{0}$, we have, by (iv), $A^{00} \subset A^{i 0}$. But $A^{i 0}=A^{i i}$ by (iii) and (v), so $A^{00} \subset A^{i i}$.

By (i), $A^{i i} \subset A^{i f}$ and, by (iv), $A^{f f} \subset A^{i f}$. Now suppose $p \notin A^{f f}$. For some $q$ in $A^{f}$ we have $|\langle p, q\rangle|=\lambda \notin^{*} K_{0}$. But then $\lambda^{\prime}=\sqrt{ } \lambda$ is also not in ${ }^{*} K_{0}$, so $1 / \lambda^{\prime} \epsilon$ ${ }^{*} K_{1}$. But then $\left(1 / \lambda^{\prime}\right) q \in A^{i}$ and $\left|\left\langle p,\left(1 / \lambda^{\prime}\right) q\right\rangle\right|=\lambda^{\prime}$, so that $p \notin A^{i f}$. This shows that $A^{i f} \subset A^{f f}$, and therefore $A^{i f}=A^{f f}$, completing the proof.

Definition 5.6. Let $A$ be a subset of ${ }^{*} E$. Fin $(A)$ is the subset of ${ }^{*} E$ defined by

$$
\operatorname{Fin}(A)=\left\{p \mid \lambda p \in A \text { for every } \lambda \in{ }^{*} K_{1}\right\}
$$

Note that, by Theorem 1.3 , if $(E, \theta)$ is a topological vector space in $\mathbb{M}$, then $\operatorname{Fin}\left(\mu_{\theta}(0)\right)=\operatorname{fin}_{\theta}(* E)$.

Lemma 5.7. If $B$ is a subset of ${ }^{*} F$, then $\mathrm{Fin}\left(B^{i}\right)=B^{f}$.

Proof. If $p \in B^{f}$ and $\lambda \in{ }^{*} K_{1}$, then $\lambda p \in B^{i}$. Therefore $B^{f} \subset \mathrm{Fin}\left(B^{i}\right)$. On the other hand, if $p \notin B^{f}$, then there exists $q \in B$ which satisfies $|\langle p, q\rangle|=\lambda \notin{ }^{*} K_{0}$. Then $1 / \lambda \in \epsilon^{*} K_{1}$ and $\left|\left\langle(1 / \lambda)_{p}, q\right\rangle\right|=1$, so that $(1 / \lambda)_{p} \notin B^{i}$. Hence $p \notin \mathrm{Fin}\left(B^{i}\right)$. This shows that $\mathrm{Fin}\left(B^{i}\right) \subset B^{f}$, and completes the proof. 
Let $\mathbb{Q}$ be a family of $\sigma(F, E)$-bounded subsets of $F$ which satisfies:

(i) $\bigcup\{A \mid A \in \mathbb{Q}\}=F$,

(ii) if $A_{1}, A_{2} \in \mathbb{Q}$, then there exists $A_{3} \in \mathbb{Q}$ such that $A_{1} \cup A_{2} \subset A_{3}$, and

(iii) if $A \in \mathbb{Q}$ and $\lambda \in K$, then $\lambda A \in \mathbb{Q}$.

In that case the family $\left\{A^{0} \mid A \in \mathbb{Q}\right\}$ of subsets of $E$ is a filter base for the neighborhood filter at 0 for a unique locally convex vector topology $\theta(\mathfrak{Q})$ on $E$. In addition, $\theta(\mathfrak{Q})$ is a Hausdorff topology and it is stronger than $\sigma(E, F)$.

Theorem 5.8. Let $\mathfrak{A}$ be as above and define $F(\mathfrak{A})=\bigcup\{* A \mid A \in \mathfrak{A}\}$. If $\theta=\theta(\mathfrak{A})$, then

$$
\mu_{\theta}(0)=F(\mathfrak{Q})^{i}=F(\mathfrak{Q})^{0} \text { and } \operatorname{fin}_{\theta}\left({ }^{*} E\right)=F(\mathfrak{Q})^{f} \text {. }
$$

Proof. Condition (iii) above on $\mathfrak{A}$ insures that $F(\mathfrak{Q})$ is closed under multiplication by elements of $N$. Therefore, $F(\mathfrak{Q})^{i}=F(\mathfrak{A})^{0}$ by Lemma 5.5(v). Also,

$$
\begin{aligned}
\mu_{\theta}(0) & =\bigcap\left\{{ }^{*}\left(A^{0}\right) \mid A \in \mathbb{Q}\right\}=\bigcap\left\{\left({ }^{*} A\right)^{0} \mid A \in \mathbb{Q}\right\} \\
& =\left(\bigcup\left\{^{*} A \mid A \in \mathbb{Q}\right\}\right)^{0}=F(\mathbb{Q})^{0}
\end{aligned}
$$

by Lemma 5.5(viii). Finally, by Theorem 1.3 and Lemma 5.7,

$$
\operatorname{fin}_{\theta}=\operatorname{Fin}\left(\mu_{\theta}(0)\right)=\operatorname{Fin}\left(F(\mathfrak{Q})^{i}\right)=F(\mathfrak{Q})^{f} .
$$

Consider the following special cases of the situation described above.

(i) $\mathfrak{Q}$ is the family of all finite subsets of $F$. Then $\theta(\mathfrak{Q})=\sigma(E, F)$ and $F(\mathfrak{Q})$ $=*[F]$. Therefore

$$
\mu_{\sigma(E, F)}(0)=\left({ }^{*}[F]\right) i \text { and } \operatorname{fin}_{\sigma(E, F)}\left({ }^{*} E\right)=\left({ }^{*}[F]\right)^{f} \text {. }
$$

(ii) $\mathcal{Q}$ is the family of all $\sigma(F, E)$-compact, absolutely convex subsets of $F$. Then $\theta(\mathfrak{Q})$ is the Mackey topology $\tau(E, F)$. By Theorem 5.8,

$$
\mu_{\tau(E, F)}(0)=F(\mathfrak{Q})^{i} \text { and } \operatorname{fin}_{\tau(E, F)}\left({ }^{*} E\right)=F(\mathfrak{Q})^{f} \text {. }
$$

(iii) $\mathfrak{Q}$ is the family of all $\sigma(F, E)$-bounded subsets of $F$. Then $\theta(\mathfrak{Q})$ is the strong topology $\beta(E, F)$ and $F(\mathfrak{Q})=\mathrm{bd}_{\sigma(F, E)}\left({ }^{*} F\right)$. Therefore

$$
\mu_{\beta(E, F)}(0)=\left(\operatorname{bd}_{\sigma(F, E)}\right)^{i} \text { and } \operatorname{fin}_{\beta(E, F)}\left({ }^{*} E\right)=\left(\operatorname{bd}_{\sigma(F, E)}\right)^{f} \text {. }
$$

Now let $\theta$ be an arbitrary Hausdorff locally convex vector topology on $E$ which satisfies $(E, \theta)^{\prime}=F$. Let $\mathcal{E}=\{A \mid A \subset F$ and $A$ is $\theta$-equicontinuous $\}$. By Theorem 5.8 (and the fact that $\theta=\theta(\xi)$ ) we have

$$
\mu_{\theta}(0)=F(\xi)^{i}=F(\xi)^{0} \text { and } \operatorname{fin}_{\theta}\left({ }^{*} E\right)=F(\xi) f \text {. }
$$

Theorem 5.9. Assume that $* \Re$ is $\kappa(\theta)^{+}$-saturated. Then $F(\mathscr{G})=\mu_{\theta}(0)^{i}=\mu_{\theta}(0)^{0}$ $=\mu_{\theta}(0)^{f}=\operatorname{fin}_{\theta}(* E)^{f}$. 
Proof. By Lemma 5.5(vi) and Theorem 5.8, $F(\xi) \subset \mu_{\theta}(0)^{0}=\mu_{\theta}(0)^{i} \subset \mu_{\theta}(0)^{f}=$ fin $\left.{ }^{*} E\right)^{f}$. Suppose $q \epsilon^{*} F \sim F(\mathcal{G})$. Let $\mathcal{U}$ be a local base at 0 for $\theta$, with the cardinality of $\mathcal{U}$ equal to $\kappa(\theta)$. Then $F(\mathcal{G})=\bigcup\left\{*\left(U^{0}\right) \mid U \in \mathcal{U}\right\}$. Therefore, for each $U$ in $\mathcal{U}$, the internal set $A(U)=\left\{p \mid p \epsilon^{*} U\right.$ and $\left.|\langle p, q\rangle| \geq 1\right\}$ is nonempty. Since $\mathcal{U}$ is a filter base it follows that the family $\{A(U) \mid U \in \mathcal{U}\}$ has the finite intersection property. From Theorem 2.7.12 of [6] it follows that there exists $p \in \bigcap\{A(U) \mid U \in \mathcal{U}\}$. That is, $p \in \mu_{\theta}(0)$ and $|\langle p, q\rangle| \geq 1$. By Theorem 1.6 there is an infinite integer $\omega$ in ${ }^{*} N$ which satisfies $\omega p \in \mu_{\theta}(0)$. Therefore, $q$ is not in $\mu_{\theta}(0)^{f}$. This shows that $\mu_{\theta}(0)^{f} \subset F(\xi)$, and completes the proof.

Definition 5.10. Let $(E, \theta)$ be a Hausdorff locally convex space in $\pi$ and let $F=(E, \theta)^{\prime}$. Let $\mathcal{E}$ be the collection of $\theta$-equicontinuous subsets of $F$. Then define

$$
M_{\theta}=F(\xi) \text { and } m_{\theta}=\operatorname{fin}_{\theta}\left({ }^{*} E\right)^{i} \text {. }
$$

Theorem 5.11. Assume $* \pi$ is $\kappa(\theta)+$-saturated.

(i) $\left.\left(m_{\theta}\right)^{i}=\left(m_{\theta}\right)^{0}=\left(m_{\theta}\right)^{f}=\operatorname{fin}_{\theta}{ }^{*} E\right)$,

(ii) $M_{\theta}=\operatorname{Fin}\left(m_{\theta}\right)$.

Proof. Since $m_{\theta}$ is of the form $A^{i}$, it follows that $\left(m_{\theta}\right)^{i}=\left(m_{\theta}\right)^{0}$ by Lemma 5.5. Also $\left(m_{\theta}\right)^{i} \subset\left(m_{\theta}\right)^{f}=\left(\text { fin }_{\theta}\right)^{i f}=\left(\text { fin }_{\theta}\right)^{f f}$ by the definition and Lemma 5.5. Moreover, fin $_{\theta} \subset\left(\text { fin }_{\theta}\right)^{i i}=\left(m_{\theta}\right)^{i}$. Theorems 5.8 and 5.9 imply that fin $\theta=\left(\mathrm{fin}_{\theta}\right)^{f f}$, which proves (i). By Lemma 5.7 and Theorem 5.9, $M_{\theta}=\left(\text { fin }_{\theta}\right)^{f}=\operatorname{Fin}\left(\left(\operatorname{fin}_{\theta}\right)^{i}\right)=\operatorname{Fin}\left(m_{\theta}\right)$ which proves (ii).

Remark. Let $(E, \theta)$ be a Hausdorff locally convex space in $M$ and suppose that $* \pi$ is $\kappa(\theta)+$-saturated. If $s$ is any finite sequence of the symbols $i, 0$ and $f$, then the previous results show how to calculate each of the sets

$$
\left(\mu_{\theta}(0)\right)^{s} \text { and }\left(\operatorname{fin}_{\theta}\right)^{s}
$$

and that the result will be one of the sets $\mu_{\theta}(0)$, fin $\theta, M_{\theta}$ or $m_{\theta}$. (It seems that this degree of saturation is necessary for the simple relationships in Theorems 5.9 and 5.11 to be true.)

Theorem 5.12. Let $(E, \theta)$ be a Hausdorff locally convex space in $\mathbb{M}$ and let $F=(E, \theta)^{\prime}$. Assume that $* \pi\left(\right.$ is $\kappa(\theta)^{+}$-saturated and $\boldsymbol{N}_{0}$-enlarging. Then $m_{\theta}$ is the monad of a filter on $F$ if and only if there is a norm on $E$ which generates the topology $\theta$.

Proof. If $\theta$ is generated on $E$ by a norm $\rho$, then $m_{\theta}$ is the set of elements of ${ }^{*} F$ which are infinitesimal relative to the dual norm $\rho^{\prime}$ on $F$. Therefore $m_{\theta}$ is the monad of the filter of neighborhoods of 0 in the normed space $\left(F, \rho^{\prime}\right)$.

Conversely, suppose $m_{\theta}=\mu(\mathfrak{F})$ for some filter $\mathcal{F}$ on $F$. If $V$ is in $\mathcal{F}$, then ${ }^{*} V \supset m_{\theta}$, and therefore, ${ }^{*} V^{0} \subset$ fin $_{\theta}$, by Theorem 5.9. If $p$ is any element of $\operatorname{fin}_{\theta}$ and $q \in m_{\theta}$, then $|\langle p, q\rangle| \leq 1$. Therefore, if we set 


$$
\mathfrak{Q}=\{W \mid W \in * \mathcal{F} \text { and }|\langle p, q\rangle| \leq 1 \text { for all } q \in W\}
$$

then $\mathfrak{Q}$ is an internal set. Moreover, $\mathfrak{Q}$ contains any element $W$ of $* \mathfrak{F}$ which satisfies $W \subset \mu(\mathfrak{F})=m_{\theta}$. It follows from Theorem 2.7.3 of [6] that there exists $V$ in $\mathcal{F}$ which satisfies ${ }^{*} V \in \mathbb{Q}$. Therefore, $p$ is in ${ }^{*} V^{0}$. Thus we have shown

$$
\operatorname{fin}_{\theta}=\bigcup\left\{^{*}\left(V^{0}\right) \mid V \in \mathcal{F}\right\} .
$$

But Theorem 2.1 implies that $V^{0}$ is $\theta$-bounded for every $V$ in $\mathcal{F}$, and therefore fin $_{\theta}=\mathrm{bd}_{\theta}$. Theorem 2.7 implies that $\theta$ is generated on $E$ by a norm, completing the proof.

Theorem 5. 13. (i) pns $\sigma(E, F)\left({ }^{*} E\right)=$ fin $_{\sigma(E, F)}\left({ }^{*} E\right)$.

(ii) For every $\phi$ in $F^{\sharp}$ there exists a $\sigma(E, F)$-finite element $p$ of ${ }^{*} E$ such that $\langle\phi, y\rangle=\langle p, * y\rangle$ for all $y$ in $F$.

Proof. Recall that fin $\sigma(E, F)=(*[F])^{f}$.

(i) By Theorem 1.2 it suffices to show that $*[E]$ is $\tilde{\theta}$-dense in fin $\theta$, where $\theta=\sigma(E, F)$. Suppose $p$ is $\sigma(E, F)$-finite and $U$ is a $\sigma(E, F)$-neighborhood of 0 . It may be assumed that $U=\left\{y_{1}, \cdots, y_{n}\right\}^{0}$ where $y_{1}, \cdots, y_{n}$ are independent over $K$. Then for each $j=1, \cdots, n,\left\langle p, *_{j}\right\rangle$ is in ${ }^{*} K_{0}$. Therefore there exists an element $x$ of $E$ which satisfies $\left\langle x, y_{j}\right\rangle=s t\left\langle p,{ }^{*} y_{j}\right\rangle$ for $j=1, \cdots, n$. From this it

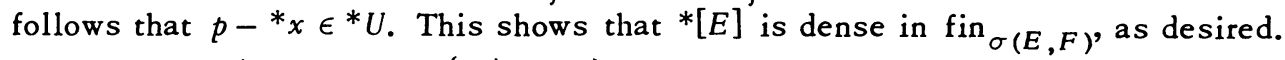

(ii) Let $\phi \in F$ and let $\left\{y_{t} \mid t \in T\right\}$ be a Hamel basis for $F$ over $K$. For each finite subset $S$ of $T$ let

$$
H(S)=\left\{x \mid x \in E \text { and }\left\langle\phi, y_{t}\right\rangle=\left\langle x, y_{t}\right\rangle \text { for all } t \in S\right\} .
$$

The collection $\{H(S) \mid S$ is a finite subset of $T\}$ is a filter base for a filter $\mathcal{F}$ on $E$. Since $* M$ is an enlargement, there is an element $p$ of $\mu(\mathcal{F})$. Then $\left\langle p,{ }^{*} y_{t}\right\rangle=$ $\langle\phi, y\rangle$ for every $t$ in $T$. From this it follows that $\langle p, * y\rangle=\langle\phi, y\rangle$ for all $y$ in $F$. In particular, $p \in(*[F])^{f}=$ fin $_{\sigma(E, F)}$, completing the proof.

From Theorem 5.13 it is immediate that the nonstandard hull of $(E, \sigma(E, F))$ is isomorphic, as a topological vector space, to $\left(F^{\#}, \sigma\left(F^{\#}, F\right)\right)$. Another simple consequence of Theorem 5.13 (using Theorem 2.4) is the following standard result.

Corollary 5.14. Every $\sigma(E, F)$-bounded subset of $E$ is $\sigma(E, F)$-totally bounded.

6. $\theta$-pre-nearstandard elements of ${ }^{*} E$ and a theorem of Grothendieck. Let $(E, \theta)$ be a Hausdorff locally convex space in $\pi$ and let $F=(E, \theta)^{\prime}$. The following result gives a characterization of the $\theta$-pre-nearstandard elements of ${ }^{*} E$ in terms of the operations and sets discussed in the previous section. Theorems 6.1 and 6.2 generalize the discussion of the pre-nearstandard points of a normed space given in $\$ 3.17$ of [6]. 
Theorem 6.1. Assume that $* \Re$ is $\boldsymbol{\aleph}_{0}$-enlarging. If $(E, \theta)$ is a Hausdorff locally convex space and $F=(E, \theta)^{\prime}$, then

$$
\operatorname{pns}_{\theta}\left({ }^{*} E\right)=\left(M_{\theta} \cap \mu_{\sigma(F, E)}(0)\right)^{i}
$$

Proof. For convenience designate $M_{\theta} \cap \mu_{\sigma(F, E)}(0)$ by $G$. It will be shown first that pns ${ }_{\theta} \subset G^{i}$. Suppose $p \in$ pns $\theta$ and $q \in G$. Then $q \in *\left(U^{0}\right)$ for some $\theta$-neighborhood $U$ of 0 . Since $p$ is $\theta$-pre-nearstandard, there exists $x \in E$ which satisfies $p-{ }^{*} x \in 1 / 2 * U$. From this and the fact that $q \in \mu_{\sigma(F, E)}(0)$, it follows that

$$
|\langle p, q\rangle| \leq\left|\left\langle p-{ }^{*} x, q\right\rangle\right|+\left|{ }^{*} x, q\right\rangle \mid<1 / 2+1 / 2=1 .
$$

Thus $p \in G^{0}$. However, $G$ is closed under multiplication by elements of $N$, so by Lemma 5.5(v), $G^{0}=G^{i}$. That is, pns ${ }_{\theta} \subset G^{i}$.

Now suppose $p \in G^{i}$. Let $U$ be an absolutely convex $\theta$-closed, $\theta$-neighborhood of 0 and let $\mathcal{O}$ be the filter of $\sigma(F, E)$-neighborhoods of 0 . Define the internal set 60 by

$$
W=\left\{V \mid V \in *^{*} \vartheta \text { and }|\langle p, q\rangle| \leq 1 \text { for each } q \in\left({ }^{*} U\right)^{0} \cap V\right\} \text {. }
$$

If $V \in * \mathcal{O}$ and $V \subset \mu(\mathcal{O})=\mu_{\sigma(F, E)}(0)$, the assumption on $p$ implies that $V \in \mathbb{O}$. Since $* M$ is $K_{0}$-enlarging, Theorem 2.7 .3 of [6] is applicable and implies that, for some $Z \in \mathcal{O}, * Z$ is in 60 . Indeed it may be assumed that $Z=S^{0}$ for some finite set $S=\left\{x_{1}, x_{2}, \cdots, x_{n}\right\}$ in $E$. So $p \in\left[*\left(U^{0} \cap S^{0}\right)\right]^{0}=*\left[\left(U^{0} \cap S^{0}\right)^{0}\right]$.

Now $U^{00}=U$ is $\sigma(E, F)$-closed and $S^{00}$ is the absolutely convex hull of the of the finite set $S$, hence $S^{00}$ is $\sigma(E, F)$-compact. Thus $U+S^{00}$ is $\sigma(E, F)$-closed and

$\left(U^{0} \cap S^{0}\right)^{0}=\sigma(E, F)$-closed absolutely convex hull of $U \cup S^{00} \subset U+S^{00}$. So $p \in \epsilon^{*} U+{ }^{*} S^{00}$. In other words, $p=u+\sum_{i=1}^{n} \lambda_{i}{ }^{*} x_{i}$ where $u \in * U, 0 \leq \lambda_{i} \in{ }^{*} R_{0}$, for each $i$, and $\sum_{i=1}^{n} \lambda_{i}=1$. Let $\delta_{i}=\operatorname{st}\left(\lambda_{i}\right)$ for $i=1,2, \ldots, n$. Then $\left(\lambda_{i}-\delta_{i}\right) *_{i} \epsilon$ $\mu_{\theta}(0) \subset * U$, so $p \in 2^{*} U+*\left(\sum_{i=1}^{n} \delta_{i} x_{i}\right)$. Thus for each $\sigma(E, F)$-closed absolutely convex $\theta$-neighborhood $U$ of $0,(p+2 * U) \cap *[E] \neq \emptyset$. It follows from Theorem 1.2 that $p \in$ pns $\theta$ Hence $G^{i} \subset$ pns $_{\theta}$ and the proof is complete.

Theorem 6.2. Let $(E, \theta)$ be a Hausdorff locally convex space and let $F=$ $(E, \theta)^{\prime}$.

(i) Assume $* \pi$ is $\aleph_{0}$-enlarging. If $g \in F^{\#}$ and $g$ is $\sigma(F, E)$-continuous on every $\theta$-equicontinuous subset of $F$, then there exists $\left.p \in \operatorname{pns} \theta^{(*} E\right)$ which satisfies $\langle g, y\rangle=\langle p, * y\rangle$ for all $y \in F$.

(ii) If $p \in \operatorname{pns}_{\theta}(* E)$ then an element $g \in F^{\#}$ is defined by $\langle g, y\rangle=s t\langle p, * y\rangle$ for all $y \in F$, and $g$ is $\sigma(F, E)$-continuous on every $\theta$-equicontinuous subset of $F$. 
Proof. Recall that a subset $A$ of $F$ is $\theta$-equicontinuous if and only if $A \subset U^{0}$ for some $\theta$-neighborhood $U$ of 0 .

(i) Suppose $g$ is $\sigma(F, E)$-continuous on each $\theta$-equicontinuous subset of $F$. Then for each $\theta$-neighborhood $U$ of 0 there exists a finite set $S(U)$ in $E$ such that $y \in U^{0} \cap S(U)^{0}$ implies $|\langle g, y\rangle| \leq 1$. It is shown below that for each finite family $\left\{U_{1}, \cdots, U_{n}\right\}$ of $\theta$-neighborhoods of 0 and each finite set $\left\{y_{1}, \cdots, y_{m}\right\}$ of elements $F$, there exists $x$ in $E$ such that

(a) $x \in\left(U_{i}^{0} \cap S\left(U_{i}\right)^{0}\right)^{0}$ for $i=1, \cdots, n$; and

(b) $\left\langle g, y_{j}\right\rangle=\left\langle x, y_{j}\right\rangle$ for $j=1, \ldots, m$.

Then since ${ }^{*} \pi$ is an enlargement it follows that there exists $p$ in ${ }^{*} E$ such that (by (a)) $p \in\left({ }^{*} U^{0} \cap * S(U)^{0}\right)^{0}$ for each $\theta$-neighborhood $U$ of 0 and (by (b)) $\langle g, y\rangle=$ $\langle p, * y\rangle$ for all $y$ in $F$. But the first of these properties implies

$$
p \in\left(M_{\theta} \cap \mu_{\sigma(E, F)}(0)\right)^{0}=\left(M_{\theta} \cap \mu_{\sigma(E, F)}(0)\right)^{i} .
$$

Thus, by Theorem $6.1, p \in$ pns $\theta$.

Now let $U_{1}, \cdots, U_{n}$ and $y_{1}, \cdots, y_{m}$ be given as above. Let $H_{0}$ be the span of $\left\{y_{1}, \ldots, y_{m}\right\}$ in $F$ and let $H_{i}=\operatorname{span} U_{i}^{0}$ for $i=1, \ldots, n$. Then $g$ is $\sigma(F, E)$-continuous on $H_{i}$ for $i=0,1, \cdots, n$, so $g$ is $\sigma(F, E)$-continuous on $H=H_{0}+H_{1}+\cdots$ $+H_{n}$. Thus by the Hahn-Banach theorem the restriction of $g$ to $H$ may be extended to an element $x$ of $(F, \sigma(F, E))^{\prime}=E$. Clearly $x$ has properties (a) and (b).

(ii) Suppose $p \in \operatorname{pns}_{\theta}$. Then $p \in$ fin $_{\theta} \subset$ fin $_{\sigma(E, F)}$, so $g \in F^{\#}$ can be defined by

$$
\langle g, y\rangle=s t\left\langle p,{ }^{*} y\right\rangle \text { for all } y \in F
$$

Let $U$ be a $\theta$-neighborhood of 0 . By Theorem 1.2 there exists $x$ in $E$ such that $p \in 1 / 2 * U+{ }^{*} x$. Thus if $y \in U^{0} \cap\{1 / 2 x\}^{0}$, then $|\langle p, * y\rangle| \leq 1 / 2+1 / 2=1$ and therefore $|\langle g, y\rangle| \leq 1$ for all $y \in U^{0} \cap\{1 / 2 x\}^{0}$. Thus $g$ is $\sigma(F, E)$-continuous on $U^{0}$ for each $\theta$-neighborhood $U$ of 0 and the proof is complete.

Lemma 6.3. Assume that $* \pi$ is $\boldsymbol{\aleph}_{0}$-enlarging. Let $(E, \theta)$ be a Hausdorff locally convex space and let $F=(E, \theta)^{\prime}$. Then

$$
\mathrm{ns}_{\theta}\left({ }^{*} E\right)=\operatorname{pns}_{\theta}\left({ }^{*} E\right) \cap \mathrm{ns}_{\sigma(E, F)}\left({ }^{*} E\right) .
$$

Proof. Clearly, $\mathrm{ns}_{\theta} \subset$ pns $_{\theta} \cap \mathrm{ns}_{\sigma(E, F)}$.

Let $p \in$ pns $_{\theta} \cap \mathrm{ns}_{\sigma(E, F)}$. Then for some $x$ in $E, p-{ }^{*} x \in \mu_{\sigma(E, F)}(0)$. Suppose $p-{ }^{*} x \notin \mu_{\theta}(0)$. Then by Theorem 5.8 there exists $q \in M_{\theta}$ such that $\left\langle p-{ }^{*} x, q\right\rangle$ $\notin^{*} K_{1}$. Now $q \in M_{\theta}$ implies $q \in \mathrm{ns}_{\sigma(F, E)}(* F)$. So for some $y$ in $F, q-{ }^{*} y \epsilon$ $\mu_{\sigma(F, E)}(0)$. Then $q-{ }^{*} y \in M_{\theta} \cap \mu_{\sigma(F, E)}(0)$ and, by Theorem 6.1, $\left\langle p, q-{ }^{*} y\right\rangle \epsilon$ ${ }^{*} K_{1}$. Since $\left\langle p-{ }^{*} x,{ }^{*} y\right\rangle \in{ }^{*} K_{1}$ and $\left\langle *^{*}, q-{ }^{*} y\right\rangle \in{ }^{*} K_{1}$ it follows that

$$
\left\langle p-{ }^{*} x, q\right\rangle=\left\langle p-{ }^{*} x,{ }^{*} y\right\rangle+\left\langle p, q-{ }^{*} y\right\rangle-\left\langle{ }^{*} x, q-{ }^{*} y\right\rangle
$$


belongs to ${ }^{*} K_{1}$ which is a contradiction. Thus $p-{ }^{*} x \in \mu_{\theta}(0)$ and $p \in \mathrm{ns} \theta$. Hence pns $_{\theta} \cap \mathrm{ns} \mathrm{s}_{\sigma(E, F)} \subset \mathrm{ns}{ }_{\theta}$ and the proof is complete.

The following completeness criterion of Grothendieck follows easily from the above results. Note that it is a consequence of Theorem 3.14.1 of [6] that a topological vector space $(E, \theta)$ is complete if and only if $\operatorname{pns}_{\theta}=\mathrm{ns} \theta$.

Theorem 6.4. Let $(E, \theta)$ be a Hausdorff locally convex space and $F=(E, \theta)^{\prime}$. Then $(E, \theta)$ is complete if and only if every element of $F^{\#}$ which is $\sigma(F, E)$-continuous on eacb $\theta$-equicontinuous subset of $F$ is in $E$.

Proof. Assume $* \mathbb{M}$ is $\boldsymbol{N}_{0}$-enlarging. Suppose $(E, \theta)$ is complete, $g \in F^{\#}$, and $g$ is $\sigma(F, E)$-continuous on each $\theta$-equicontinuous subset of $F$. Then by Theorem 6.2 there exists $p \in$ pns $_{\theta}=$ ns $_{\theta}$ such that $\langle g, y\rangle=\left\langle p,{ }^{*} y\right\rangle$ for all $y$ in $F$. But for some $x$ in $E, p-{ }^{*} x \in \mu_{\theta}(0) \subset \mu_{\sigma(E, F)}(0)$. It follows that $\left\langle p,{ }^{*} y\right\rangle={ }_{1}\langle x, y\rangle$ for all $y$ in $F$. Thus $g=x \in E$.

Now suppose that whenever $g \in F^{\#}$ and $g$ is $\sigma(F, E)$-continuous on each $\theta$ equicontinuous subset of $F$, then $g \in E$. Let $p \in$ pns $\theta$. By Theorem 6.2 the element $x$ of $F^{\#}$ defined by $\langle x, y\rangle=$ st $\langle p, * y\rangle$ for $y$ in $F$ is $\sigma(F, E)$-continuous on each $\theta$-equicontinuous subset of $F$. Thus $x \in E$ and $p-{ }^{*} x \in \mu_{\sigma(E, F)}(0)$. Hence

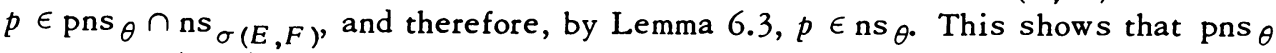
$=\mathrm{ns} \theta$ and $(E, \theta)$ is complete.

Theorem 6.5. Let $(E, \theta)$ be a complete Hausdorff locally convex space. If $\Delta$ is a locally convex vector topology on $E$ which satisfies $\theta \subset \Delta$ and $(E, \Delta)^{\prime}=$ $(E, \theta)^{\prime}$, then $(E, \Delta)$ is also complete.

Proof. Evidently, pns ${ }_{\Delta} \subset$ pns $_{\theta}=\mathrm{ns}_{\theta} \subset \mathrm{ns}_{\sigma(E, \dot{F})}$, where $F=(E, \theta)^{\prime}=(E, \Delta)^{\prime}$. Therefore, by Theorem 6.3, pns ${ }_{\Delta}=$ pns $_{\Delta} \cap n s_{\sigma(E, F)}=n s_{\Delta}$, and $(E, \Delta)$ is complete.

7. A theorem of Krein.

Lemma 7.1. Assume that $* M$ is $\aleph_{1}$-saturated. Let $\mathfrak{B}$ be the Boolean algebra of all internal subsets of $I_{\omega}=\{n \mid 1 \leq n \leq \omega\}$ where $\omega \epsilon^{*} N$, and let $\mu$ be any nonnegative, finitely additive measure on $\mathfrak{B}$.

If $\left\{A_{n} \mid n \in N\right\}$ is a sequence of internal subsets of $I_{\omega}$ which satisfies $\lim \inf A_{n}=I_{\omega}$, then $\lim _{n} \mu\left(A_{n}\right)=\mu\left(I_{\omega}\right)$.

Proof. If $\mu\left(I_{\omega}\right)=0$, then the result is trivial. Thus we may assume $\mu\left(I_{\omega}\right)=1$. If the lemma fails for a sequence $\left\{A_{n}\right\}$, then by selecting a subsequence if necessary, it may be assumed that there exists $\delta>0$ in $R$ which satisfies $\mu\left(I_{\omega} \sim A_{n}\right)$ $\geq \delta$ for all $n$ in $N$. It follows that we may select a sequence $n_{1}<n_{2}<\ldots$ in $N$ such that

$$
\bigcap\left\{I_{\omega} \sim A_{n_{j}} \mid 1 \leq j \leq r\right\} \neq \varnothing
$$


for each $r$ in $N$. Since $* M$ is $\aleph_{1}$-saturated, Theorem 2.7.12 of [6] implies that

$$
\bigcap\left\{I_{\omega} \sim A_{n_{j}} \mid j \in N\right\} \neq \varnothing,
$$

which contradicts the assumption that $\lim \inf A_{n}=I_{\omega}$.

Theorem 7.2. Assume $* \mathbb{M}$ is $\boldsymbol{\aleph}_{1}$-saturated. Let $(E, \theta)$ be a topological vector space, $A$ a $\theta$-compact subset of $E, C$ the convex bull of $A$, and $\left\{f_{n} \mid n \in N\right\}$ a sequence of continuous linear functionals on $E$ which are uniformly bounded on $A$. If $\lim _{n} f_{n}(x)=0$ for every $x \in A$ and if $p \in * C$, then $\lim _{n}$ st $\left(* f_{n}(p)\right)=0$.

Proof. It may be assumed that $\left|f_{n}(x)\right| \leq 1$ for every $x$ in $A$ and every $n$ in $N$. Therefore $\left|* f_{n}(q)\right| \leq 1$ for $q$ in $* A$ and $n$ in $N$. Also if $q \in \epsilon^{*} A$ then there exists $x$ in $A$ which satisfies $q-* x \in \mu_{\theta}(0)$. Since each $f_{n}$ is continuous, ${ }^{*} f_{n}(q)={ }_{1}$ $f_{n}(x)$. Therefore $q \in \epsilon^{*} A$ implies $\lim _{n}$ st $\left(* f_{n}(q)\right)=0$.

Now let $p$ be an element of ${ }^{*} C$. Since ${ }^{*} C$ is the absolutely hyperconvex hull of $* A$, there exist $\omega \in \epsilon^{*} N$, an internal sequence $\left\{q_{1}, q_{2}, \cdots, q_{\omega}\right\}$ of elements of $* A$ and an internal sequence $\left\{\lambda_{1}, \lambda_{2}, \ldots, \lambda_{\omega}\right\}$ of elements of ${ }^{*} R^{+}$which satisfy $\sum_{k=1}^{\omega} \lambda_{k}=1$ and $p=\sum_{k=1}^{\omega} \lambda_{k} q_{k}$.

Let $\delta>0$ be in $R$. For each $n$ in $N$ define

$$
A_{n}=\left\{k \mid 1 \leq k \leq \omega \text { and }\left|f_{n}\left(q_{k}\right)\right|<\delta / 2\right\} \text {. }
$$

It follows that $\lim \inf A_{n}=I_{\omega}=\{1,2, \cdots, \omega\}$. A nonnegative, finitely additive measure $\mu$ may be defined on the Boolean algebra of internal subsets $A$ of $I_{\omega}$ by

$$
\mu(A)=\operatorname{st}\left(\sum_{k \in A} \lambda_{k}\right) .
$$

Then $\mu\left(I_{\omega}\right)=1$, so by Lemma 7.1 there exists $n_{0}$ such that $n \geq n_{0}$ implies $\mu\left(A_{n}\right)>$ $1-\delta / 2$ and therefore $\Sigma_{k \in I_{\omega} \sim A_{n}} \lambda_{k}<\delta / 2$. Then for $n \geq n_{0}$, we have

$$
\begin{aligned}
\left|f_{n}(p)\right| & =\left|\sum_{k=1}^{\omega} \lambda_{k}{ }^{*} f_{n}\left(q_{k}\right)\right| \leq\left.\sum_{k \in A_{n}} \lambda_{k}\right|^{*} f_{n}\left(q_{k}\right)\left|+\sum_{k \in I_{\omega} \sim A_{n}} \lambda_{k}\right|^{*} f_{n}\left(q_{k}\right) \mid \\
& \leq \frac{\delta}{2} \sum_{k=1}^{\omega} \lambda_{k}+\sum_{k \in I_{\omega} \sim A_{n}} \lambda_{k}<\frac{\delta}{2}+\frac{\delta}{2}=\delta .
\end{aligned}
$$

Since $\delta>0$ was an arbitrary element of $R^{+}$, this shows that $\lim _{n}$ st $\left(* f_{n}(p)\right)=0$.

An immediate consequence of Theorem 7.2 is the following standard result (Theorem 17.11 in [5]).

Corollary 7.3. Let $(E, \theta)$ be a topological vector space, $A$ a $\theta$-compact subset of $E$, and $\left\{f_{n}\right\}$ a sequence of continuous linear functionals on $E$ which are uniformly bounded on $A$.

If $\lim _{n} f_{n}(x)=0$ for every $x \in A$ and if $z$ is in the closed convex bull of $A$, then $\lim _{n} f_{n}(z)=0$. 
Proof. Assume that $* \pi$ is $\aleph_{1}$-saturated and $\pi$ has $(E, \theta)$ as an element. Let $C$ be the convex hull of $A$. If $z$ is in the closure of $C$, then for some $p$ in ${ }^{*} C$ we have $p-{ }^{*} z \in \mu_{\theta}(0)$. Therefore, $f_{n}(z)=s t\left(f_{n}(p)\right)$ for every $n$ in $N$, and Theorem 7.2 implies that $\lim _{n} f_{n}(z)=0$.

We now give a nonstandard proof of a theorem due to Krein.

Theorem 7.4. Let $(E, \theta)$ be a complete Hausdorff locally convex space and let $F=(E, \theta)^{\prime}$. If $A$ is a $\sigma(E, F)$-compact subset of $E$, then the $\sigma(E, F)$-closed convex bull of $A$ is again $\sigma(E, F)$-compact.

Proof. Assume that $* \pi$ is $\aleph_{1}$-saturated and $\pi$ has $(E, \theta)$ as an element. Let $C$ be the convex hull of $A$. By Theorem 3.6.2 of [6] it suffices to show that every element of ${ }^{*} C$ is $\sigma(E, F)$-nearstandard to an element of $E$. Since $A$ is $\sigma(E, F)$ compact, $C$ is $\sigma(E, F)$-bounded and therefore every element of ${ }^{*} C$ is $\sigma(E, F)$-finite, by Theorem 2.1.

Let $p \in{ }^{*} C$. An element $w$ of $F^{\#}$ may be defined by

$$
\langle w, y\rangle=\operatorname{st}\left\langle p,{ }^{*} y\right\rangle
$$

for all $y$ in $F$. It remains only to show that $w \in E$. If not, then it follows from Grothendieck's completeness criterion (Theorem 6.4 ) that there is a $\theta$-neighborhood $U$ of 0 such that $w$ is not $\sigma(F, E)$-continuous at 0 on $U^{0}$. That is, for some $\delta>$ 0 in $R$, if $\eta>0$ is in $R$ and $S$ is a finite subset of $E$, then there exists $y \in U^{0}$ which satisfies $|\langle x, y\rangle|\langle\eta$ for all $x$ in $S$, but $|\langle w, y\rangle|>\delta$.

We now define a sequence $\left\{y_{n} \mid n \in N\right\}$ of elements of $U^{0}$ and a sequence $\left\{D_{n} \mid\right.$ $n \in N\}$ of finite or countable subsets of $A$. Each set $D_{n}$ will be enumerated (possibly with repetitions) as $D_{n}=\{d(n, 1), d(n, 2), \ldots\}$. First choose $y_{1} \in U^{0}$ so that $\left.\left|\left\langle w, y_{1}\right\rangle\right|\right\rangle \delta$. Choose $D_{1} \subset A$ so that given $x$ in $A$ and $\eta>0$ in $R$ there exists $d \in D_{1}$ which satisfies $\left|\left\langle x-d, y_{1}\right\rangle\right|<\eta$. ( $D_{1}$ may be taken to be finite or countable since $A$ is $\sigma(F, E)$-compact.) Proceeding by induction, assume $y_{1}, \cdots, y_{n}$ and $D_{1}, \cdots, D_{n}$ have been chosen. Select $y_{n+1} \in U^{0}$ so that $\left|\left\langle w, y_{n+1}\right\rangle\right|>\delta$ but, for each $i, j \geq 1$ with $i+j \leq n+1,\left|\left\langle d(i, j), y_{n+1}\right\rangle\right| \leq 2^{-n}$. Then choose a finite or countable subset $D_{n+1}$ of $A$ such that for each $x$ in $A$ and $\eta>0$ in $R$, there exists $d$ in $D_{n+1}$ with $\left|\left\langle x-d, y_{j}\right\rangle\right|<\eta$ for all $j=1, \cdots, n+1$. Thus the sequences $\left\{y_{n}\right\}$ and $\left\{D_{n}\right\}$ satisfy

(i) $\left|\left\langle w, y_{n}\right\rangle\right|>\delta$ for all $n \in N$;

(ii) if $d \in \bigcup\left\{D_{k} \mid k \in N\right\}$, then $\lim _{n}\left\langle d, y_{n}\right\rangle=0$;

(iii) if $x \in A, \eta>0$ is in $R$, and $n \in N$, then for some $d \in D_{n}$ we have

$$
\left|\left\langle x-d, y_{j}\right\rangle\right|<\eta \quad \text { for } 1 \leq j \leq n \text {. }
$$

Let $D=\bigcup\left\{D_{k} \mid k \in N\right\}$. It will be shown that $\lim _{n}\left\langle x, y_{n}\right\rangle=0$ for every $x$ in $A$. If this fails for some $x$ in $A$, then there is an infinite integer $\omega$ in ${ }^{*} N$ such 
that $\left\langle * x, * y_{\omega}\right\rangle \notin^{*} K_{1}$. But $* y_{\omega}$ is $\sigma(F, E)$-nearstandard to some $y_{0}$ in $F$, since $U^{0}$ is $\sigma(F, E)$-compact. Condition (ii) above implies that $\left\langle * d,{ }^{*} y_{\omega}\right\rangle \in{ }^{*} K_{1}$ for every $d \in D$. Thus $\left\langle d, y_{0}\right\rangle=0$ for all $d \in D$. It follows that $d \in{ }^{*} D$ implies $\langle d$, $\left.* y_{0}\right\rangle=0$. Passing condition (iii) above to $* \Re$ (with $\eta \in{ }^{*} K_{1}$ and $n=\omega$ ), there exists $d \in \epsilon^{*} D$ with $\left\langle{ }^{*} x-d,{ }^{*} y_{j}\right\rangle \in *^{*} K_{1}$ for every $1 \leq j \leq \omega$.

Let $d_{0}$ be the $\sigma(E, F)$-standard part of $d$, so that $\left\langle x-d_{0}, y_{j}\right\rangle=0$ for every $j$ in $N$. Therefore, $\left\langle * x-{ }^{*} d_{0}, y_{\omega}\right\rangle=0$, and hence

$$
\left\langle{ }^{*} x,{ }^{*} y_{\omega}\right\rangle=\left\langle{ }^{*} d_{0},{ }^{*} y_{\omega}\right\rangle={ }_{1}\left\langle{ }^{*} d_{0},{ }^{*} y_{0}\right\rangle={ }_{1}\left\langle d,{ }^{*} y_{0}\right\rangle=0
$$

This contradiction shows that $\lim _{n}\left\langle x, y_{n}\right\rangle=0$ for every $x$ in $A$. Also, since $U$ absorbs $A$, the sequence $\left\{y_{n}\right\}$ is uniformly bounded on $A$.

It follows from Theorem 7.2 that $\lim _{n} s t\left(\left\langle p, y_{n}\right\rangle\right)=0$. In particular, for some $n \in N,\left|\left\langle p,{ }^{*} y_{n}\right\rangle\right|<\delta / 2$. But this implies $\left|\left\langle w, y_{n}\right\rangle\right| \leq \delta / 2$, which is a contradiction. That is, $w$ is in $E$ and the proof is complete.

8. Nonstandard hulls of normed spaces. Let $(E, \rho)$ be a normed space in $\pi$. Let fin $\rho$ be the set of $\rho$-finite elements of $* E,(\hat{E}, \hat{\rho})$ the nonstandard hull of $(E, \rho)$, and $\pi$ : fin $\rho \rightarrow \hat{E}$ the canonical surjection. (See the remarks following Definition 1.7.) The dual space $\left(E^{\prime}, \rho^{\prime}\right)$ of $(E, \rho)$ is then in $M$. Therefore we also have the nonstandard hull $\left(\widehat{E^{\prime}}, \widehat{\rho^{\prime}}\right)$ of $\left(E^{\prime}, \rho^{\prime}\right)$ and the canonical surjection $\pi^{\prime}$ : fin $\rho^{\prime} \rightarrow \widehat{E^{\prime}}$.

For $p \epsilon^{*} E$ and $q \epsilon^{*} E^{\prime}$, we have $|\langle p, q\rangle| \leq{ }^{*} \rho(p)^{*} \rho^{\prime}(q)$. It follows that if $x \epsilon$ $\hat{E}$ and $y \in \widehat{E^{\prime}}$, then we may define $\langle x, y\rangle=s t\langle p, q\rangle$ where $x=\pi(p)$ and $y=\pi^{\prime}(q)$. This defines a bilinear functional on $\hat{E} \times \widehat{E^{\prime}}$. The following result shows that this defines a pairing between $\hat{E}$ and $\widehat{E^{\prime}}$, and that, with respect to this pairing, $\left(\widehat{E^{\prime}}, \widehat{\rho^{\prime}}\right)$ is a norm subspace of the dual space $(\hat{E}, \hat{\rho})^{\prime}$, furnished with the dual norm $\hat{\rho}^{\prime}$.

Lemma 8. 1. (i) If $x \in \hat{E}$ and $y \in \widehat{E^{\prime}}$, then $|\langle x, y\rangle| \leq \hat{\rho}(x) \widehat{\rho^{\prime}}(y)$. $\widehat{\rho^{\prime}}(y)$.

(ii) If $y \in \widehat{E^{\prime}}$, then there exists $x \in \hat{E}$ which satisfies $\hat{\rho}(x)=1$ and $\langle x, y\rangle=$

Proof. (i) Let $x \in \hat{E}$ and $y \in \widehat{E^{\prime}}$ and choose $p \in \epsilon^{*} E$ and $q \epsilon^{*} E^{\prime}$ such that $x=\pi(p)$ and $y=\pi^{\prime}(q)$. Then

$$
|\langle x, y\rangle|=|s t\langle p, q\rangle| \leq \operatorname{st}\left(^{*} \rho(p)^{*} \rho^{\prime}(q)\right)=\hat{\rho}(x) \widehat{\rho^{\prime}}(y) .
$$

(ii) Let $y \in \widehat{E^{\prime}}$ and choose $q \in \epsilon^{*} E^{\prime}$ such that $y=\pi^{\prime}(q)$. Then there exists $p$ in ${ }^{*} E$ such that ${ }^{*} \rho(p)=1$ and $\langle p, q\rangle={ }_{1}{ }^{*} \rho^{\prime}(q)$, since $\rho^{\prime}$ is the dual norm on $(E, \rho)^{\prime}$. If $x=\pi(p)$, then $\hat{\rho}(p)=1$ and $\left.\langle x, y\rangle=\operatorname{st}\langle p, q\rangle=\operatorname{st}^{*} \rho^{\prime}(q)\right)=\widehat{\rho^{\prime}}(y)$.

Lemma 8.2. If $\phi$ is a $\hat{\rho}$-bounded linear functional on $\hat{E}$ and if $p_{1}, \cdots, p_{n}$ are elements of fin ${ }^{(* E)}$ such that $\left\{\pi\left(p_{1}\right), \ldots, \pi\left(p_{n}\right)\right\}$ is independent in $\hat{E}$, then there exists $q \epsilon^{*} E^{\prime}$ such that 
(i) $\left\langle p_{i}, q\right\rangle=\left\langle\pi\left(p_{i}\right), \phi\right\rangle$ for $i=1,2, \cdots, n$;

(ii) st $\left(^{*} \rho^{\prime}(q)\right) \leq \hat{\rho}^{\prime}(\phi)$.

Proof. By Theorem 1.8, $\left\{p_{1}, \cdots, p_{n}\right\}$ is ${ }^{*}$-independent over ${ }^{*} K$. Let $H$ be the

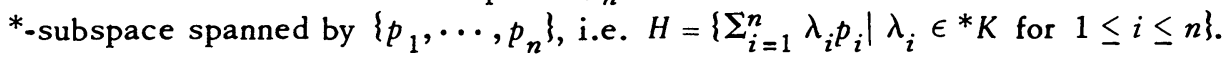
Define a $*_{-}$-linear functional $q_{0}$ on $H$ by setting

$$
q_{0}\left(\sum_{i=1}^{n} \lambda_{i} p_{i}\right)=\sum_{i=1}^{n} \lambda_{i}\left\langle\pi\left(p_{i}\right), \phi\right\rangle .
$$

Again by Theorem 1.8 , if $p=\sum_{i=1}^{n} \lambda_{i} p_{i} \in H$ and ${ }^{*} \rho(p) \leq 1$, then $\lambda_{i} \in{ }^{*} K_{0}$ for $1 \leq i$ $\leq n$ and $\pi(p)=\sum_{i=1}^{n}$ st $\left(\lambda_{i}\right) \pi\left(p_{i}\right)$. But $\hat{\rho}(\pi(p)) \leq 1$ implies

$$
\left|\left\langle\sum_{i=1}^{n} s t\left(\lambda_{i}\right) \pi\left(p_{i}\right), \phi\right\rangle\right|=s t\left|\sum_{i=1}^{n} \lambda_{i}\left\langle\pi\left(p_{i}\right), \phi\right\rangle\right| \leq \hat{\rho}^{\prime}(\phi) .
$$

Thus $q_{0}$ is ${ }^{*} \rho$-bounded on $H$ and for some $0 \leq \delta \in *^{*} K_{1}$ the ${ }^{*}$-dual norm of $q_{0}$ is bounded above by $\hat{\rho}^{\prime}(\phi)+\delta$. By passing the Hahn-Banach theorem to * $)$ it follows that there exists $q$ in ${ }^{*} E^{\prime}$ such that the restriction of $q$ to $H$ is $q_{0}$ and ${ }^{*} \rho^{\prime}(q) \leq$ $\hat{\rho}^{\prime}(\phi)+\delta$. Then $q$ satisfies (i) and (ii).

Theorem 8.3. Assume that $* M$ is $\kappa$-saturated. If $S$ is a subspace of $E$ of dimension less than $\kappa$ and if $\phi$ is a $\hat{\rho}$-bounded linear functional on $\hat{E}$, then there exists $y$ in $\widehat{E}^{\prime}$ such that $y$ agrees with $\phi$ on $S$ and $\widehat{\rho^{\prime}}(y) \leq \hat{\rho}^{\prime}(\phi)$.

Proof. Assume $S$ is nontrivial. Let $\left\{p_{i} \mid i \in I\right\}$ be a family of elements of ${ }^{*} E$ such that $\left\{\pi\left(p_{i}\right) \mid i \in I\right\}$ is a Hamel basis for $S$; thus the cardinality of $I$ is less than $\kappa$. For each finite subset $A$ of $I$ and each integer $n$ in $N$ let $T(A, n)=$ $\left\{q \in{ }^{*} E^{\prime} \mid\left\langle\pi\left(p_{i}\right), \phi\right\rangle=\left\langle p_{i}, q\right\rangle\right.$ for $i$ in $A$ and $\left.{ }^{*} \rho^{\prime}(q) \leq \hat{\rho}^{\prime}(\phi)+n^{-1}\right\}$. Then each set $T(A, n)$ is internal and by Lemma 8.2 each $T(A, n)$ is nonempty. Moreover, $\{T(A, n) \mid A$ is a finite subset of $I$ and $n \in N\}$ has cardinality less than $\kappa$ and possesses the finite intersection property. (Recall that $\kappa$ is uncountable by assumption.) By Theorem 2.7.12 of [6] there exists $q \epsilon^{*} E^{\prime}$ such that $q \in T(A, n)$ for all $A$ and $n$. Then $y=\pi^{\prime}(q)$ is the desired element of $\widehat{E^{\prime}}$.

Theorem 8.3 gives the following information about the pairing between $\hat{E}$ and $\widehat{E^{\prime}}$.

Corollary 8.4. Assume that $* \Re$ is $\aleph_{1}$-saturated. A subset of $\hat{E}$ is $\sigma\left(\hat{E}, \widehat{E^{\prime}}\right)$ bounded if and only if it is $\hat{\rho}$-bounded.

Proof. By Lemma $8.1, \widehat{E}$ is a subspace of $\hat{E}^{\prime}$. Thus every $\hat{\rho}$-bounded subset of $\hat{E}$ is $\sigma\left(\hat{E}, \widehat{E^{\prime}}\right)$-bounded.

Suppose $B$ is a $\sigma\left(\hat{E}, \widehat{E}^{\prime}\right)$-bounded subset of $\hat{E}$. If $B$ is not $\hat{\rho}$-bounded, then there exist $\phi \in \hat{E}^{\prime}$ and a countable set $\left\{x_{1}, x_{2}, \ldots\right\}$ in $B$ such that $\left|\left\langle x_{n}, \phi\right\rangle\right| \geq n$ 
for $n=1,2, \ldots$. If $S$ is the subspace spanned by $\left\{x_{1}, x_{2}, \ldots\right\}$, by Theorem 8.3 there exists $y$ in $\widehat{E^{\prime}}$ such that $y=\phi$ on $S$. But then $\left|\left\langle x_{n}, y\right\rangle\right| \geq n$ for $n=1,2, \ldots$, which contradicts the fact that $B$ is $\sigma\left(\hat{E}, \widehat{E}^{\prime}\right)$-bounded. Thus $B$ is $\hat{\rho}$-bounded.

Recall that if $* \pi$ is $\aleph_{1}$-saturated and $(E, \rho)$ is a normed space in $\pi$ then $(\hat{E}, \hat{\rho})$ is a Banach space. (See $\S 1$.) Under this saturation assumption, Theorem 8.5 (below) characterizes those normed spaces $(E, \rho)$ such that $\left(\widehat{E}^{\prime}, \widehat{\rho^{\prime}}\right)$ is the dual space of $(\hat{E}, \hat{\rho})$. The proof uses the following characterization of reflexive real Banach spaces which is due to R. C. James [4].

Theorem [James]. A real Banach space $(E, \rho)$ is nonreflexive if and only if for each number $r<1$ there exist a sequence $\left\{x_{i}\right\}$ in $E$ and a sequence $\left\{y_{j}\right\}$ in $E^{\prime}$ such that $\rho\left(x_{i}\right)=1$ and $\rho^{\prime}\left(y_{j}\right)=1$ for $i, j=1,2, \ldots ; r\left\langle\left\langle x_{i}, y_{j}\right\rangle\right.$ if $j \leq i$; and $0=$ $\left\langle x_{i}, y_{j}\right\rangle$ if $\left.j\right\rangle i$.

This result has the following analogue for complex Banach spaces $(E, \rho)$ : the space $(E, \rho)$ is nonreflexive if and only if for each real number $r<1$ there exist a sequence $\left\{x_{i}\right\}$ in $E$ and a sequence $\left\{y_{j}\right\}$ in $E^{\prime}$ such that $\rho\left(x_{i}\right)=1$ and $\rho^{\prime}\left(y_{j}\right)=1$ for $i, j=1,2, \cdots ; r<\operatorname{Re}\left\langle x_{i}, y_{j}\right\rangle$ if $j \leq i$; and $0=\operatorname{Re}\left\langle x_{i}, y_{j}\right\rangle$ if $j>i$. This is an immediate consequence of James' result, using the fact the dual space of $(E, \rho)$ as a real normed space is exactly the space of all real valued linear functions $\phi$ defined by

$$
\phi(x)=\operatorname{Re}\langle x, y\rangle, \quad x \in E,
$$

where $y$ is an arbitrary element of $E^{\prime}$. (Recall that the dual norm of $\phi$ will be equal to the dual norm of $y$ in case this equation holds on $E$.)

Theorem 8.5. Assume $* \mathbb{M}$ is $\boldsymbol{N}_{1}$-saturated and $(E, \rho)$ is a normed space in $\mathbb{M}$. The following conditions are equivalent.

(i) The dual space of $(\hat{E}, \hat{\rho})$ is $\left(\widehat{E^{\prime}}, \widehat{\rho^{\prime}}\right)$.

(ii) $(\hat{E}, \hat{\rho})$ is reflexive.

Moreover, if $(E, \rho)$ is a real normed space then these conditions are equivalent to

(iii) For some $r \in R, 0<r<1$, and some $n$ in $N$ there do not exist finite sequences $\left\{x_{1}, \cdots, x_{n}\right\}$ in $E$ and $\left\{y_{1}, \cdots, y_{n}\right\}$ in $E^{\prime}$ which satisfy $\rho\left(x_{i}\right)=1$, $\rho^{\prime}\left(y_{j}\right)=1$ for $i, j=1,2, \ldots, n ; r<\left\langle x_{i}, y_{j}\right\rangle$ if $1 \leq j \leq i \leq n$; and $0=\left\langle x_{i}, y_{j}\right\rangle$ if 1 $\leq i<j \leq n$.

If $(E, \rho)$ is a complex normed space then (i) and (ii) are equivalent to

(iii') For some $r \in R, 0<r<1$, and some $n$ in $N$ there do not exist finite sequences $\left\{x_{1}, \cdots, x_{n}\right\}$ in $E$ and $\left\{y_{1}, \cdots, y_{n}\right\}$ in $E^{\prime}$ which satisfy $\rho\left(x_{i}\right)=1, \rho^{\prime}\left(y_{j}\right)=$ 1 for $i, j=1,2, \cdots, n ; r<\operatorname{Re}\left\langle x_{i}, y_{j}\right\rangle$ if $1 \leq j \leq i \leq n$; and $0=\operatorname{Re}\left\langle x_{i}, y_{j}\right\rangle$ if $1 \leq$ $i<j \leq n$. 
Proof. We give the proof assuming $(E, \rho)$ is a real space. The proof when $(E, \rho)$ is a complex space is similar.

(i) implies (iii). Suppose that the dual space of $(\hat{E}, \hat{\rho})$ is $\left(\widehat{E^{\prime}}, \widehat{\rho^{\prime}}\right)$ but that (iii) fails. Let $r \in *^{*}$ be such that $r<1$ and $r={ }_{1} 1$, and let $\omega \in *^{*} N \sim N$. Then since the negation of (iii) holds in $* \pi$ there exist $*$ finite sequences $\left\{p_{n} \mid 1 \leq n \leq\right.$ $\omega\}$ in $* E$ and $\left\{q_{n} \mid 1 \leq n \leq \omega\right\}$ in ${ }^{*} E^{\prime}$ such that ${ }^{*} \rho\left(p_{n}\right)=1,{ }^{*} \rho^{\prime}\left(q_{m}\right)=1$ for $1 \leq n$, $m \leq \omega ; r<\left\langle p_{n}, q_{m}\right\rangle$ for $1 \leq m \leq n \leq \omega$; and $0=\left\langle p_{n}, q_{m}\right\rangle$ for $1 \leq n<m \leq \omega$. Now the closed unit ball in $\left(\hat{E}^{\prime}, \hat{\rho}^{\prime}\right)$ is $\sigma\left(\hat{E}^{\prime}, \hat{E}\right)$-compact, so there is an element $\phi$ of $\hat{E}^{\prime}$ which is a $\sigma\left(\hat{E}^{\prime}, \hat{E}\right)$-limit point of the sequence $\left\{\pi^{\prime}\left(q_{m}\right) \mid m \in N\right\}$. Then for each $1 \leq n \leq \omega$ and each $m \in N$,

$$
\left\langle\pi\left(p_{n}\right), \pi^{\prime}\left(q_{m}\right)\right\rangle= \begin{cases}0 & \text { if } m>n, \\ 1 & \text { if } m \leq n .\end{cases}
$$

It follows that

$$
\left\langle\pi\left(p_{n}\right), \phi\right\rangle= \begin{cases}0 & \text { if } n \in N \\ 1 & \text { if } n \in \epsilon^{*} N \sim N \text { and } n \leq \omega .\end{cases}
$$

But if $\phi$ is in $\widehat{E^{\prime}}$ then $\phi=\pi^{\prime}(q)$ for some $q \epsilon^{*} E^{\prime}$. Hence the set $\{n \mid 1 \leq n \leq \omega$ and $\left\langle p_{n}, q\right\rangle\langle 1 / 2\}$ is internal and equals $N$, which is a contradiction. Thus $\phi$ is not in $\overparen{E}^{\top}$ which contradicts (i). Therefore (i) implies (iii).

(iii) implies (ii). Assume that (ii) fails, so that $(\hat{E}, \hat{\rho})$ is not reflexive. Let $r$ be a real number such that $r<1$. By the theorem of James stated above, there exist sequences $\left\{x_{i} \mid i \in N\right\}$ in $\hat{E}$ and $\left\{y_{j} \mid j \in N\right\}$ in $\hat{E}^{\prime}$ such that $\hat{\rho}\left(x_{i}\right)=1, \hat{\rho}^{\prime}\left(y_{j}\right)$ $=1$ for $i, j \in N ; r\left\langle\left\langle x_{i}, y_{j}\right\rangle\right.$ if $j \leq i$; and $0=\left\langle x_{i}, y_{j}\right\rangle$ if $\left.j\right\rangle i$. For each $i$ in $N$ pick $p_{i}$ in ${ }^{*} E$ such that $\pi\left(p_{i}\right)=x_{i}$ and $*^{*}\left(p_{i}\right)=\hat{\rho}\left(x_{i}\right)=1$. By Theorem 8.3, for each $j$ in $N$ there exists $q_{j}$ in ${ }^{*} E^{\prime}$ such that $\pi^{\prime}\left(q_{j}\right)$ agrees with $y_{j}$ on $\left\{x_{i} \mid i \in N\right\}$ and st $\left({ }^{*} \rho^{\prime}\left(q_{j}\right)\right) \leq \hat{\rho}^{\prime}\left(y_{j}\right)=1$. Indeed the $q_{j}$ 's may be selected so that ${ }^{*} \rho^{\prime}\left(q_{j}\right) \leq 1$ for.each $j$ in $N$ (multiplying each $q_{j}$ by an element $\lambda_{j}$ of ${ }^{*} R$ which satisfies $\lambda_{j}={ }_{1} 1$, if necessary). Then for $n \in N,{ }^{*} \rho\left(p_{i}\right) \leq 1,{ }^{*} \rho^{\prime}\left(q_{j}\right) \leq 1$ for $1 \leq i, j \leq n ; r<\left\langle p_{i} q_{j}\right\rangle$ if $1 \leq$ $j \leq i \leq n$; and $0=\left\langle p_{i}, q_{j}\right\rangle$ if $1 \leq i<j \leq n$. But for each standard real number $r<1$ and each $n$ in $N$, this may be passed back to $M$. It follows that (iii) fails, completing the proof that (iii) implies (ii).

(ii) implies (i). If $\left(\widehat{E^{\prime}}, \widehat{\rho^{\prime}}\right)$ is not equal to $(\hat{E}, \hat{\rho})^{\prime}=\left(\hat{E}^{\prime}, \hat{\rho}^{\prime}\right)$, then by the HahnBanach theorem there is a nontrivial $\hat{\rho}^{\prime}$-bounded linear functional $\phi$ on $\hat{E}^{\prime}$ which is 0 on $\widehat{E^{\prime}}$. But if $(\hat{E}, \hat{\rho})$ is reflexive, then $\phi$ is in $\hat{E}$, which is impossible. Thus (ii) implies (i), and the proof is complete.

Let $(E, \rho)$ be a Banach space. If $(\hat{E}, \hat{\rho})$ is reflexive, then $(E, \rho)$ must also be reflexive since it is a closed subspace of $(\hat{E}, \hat{\rho})$. However, the converse of this fact does not hold, as the following example shows. 
Example 8.6. Let $\left\{p_{n} \mid n \in N\right\}$ be a strictly increasing sequence of real numbers such that $1<p_{n}$ for each $n$ and $p_{n} \uparrow \infty$. For each $n$ let $q_{n}$ be defined by $1 / p_{n}+$ $1 / q_{n}=1$. Let $E_{n}$ be the sequence space $l_{p_{n}}$ over $K$ and let $\rho_{n}$ denote the $l_{p_{n}}$ norm, for each $n$ in $N$. Then $\left(E_{n}^{\prime}, \rho_{n}^{\prime}\right)$ is $l_{q_{n}}$ with the $l_{q_{n}}$ norm.

Let $E$ be the subspace of the product $\prod_{n}^{n} E_{n}(n \in N)$ consisting of all $x=$ $\left(x_{1}, x_{2}, \ldots\right)$ such that

$$
\rho(x)=\left(\sum_{n=1}^{\infty}\left[\rho_{n}\left(x_{n}\right)\right]^{2}\right)^{1 / 2}<\infty .
$$

$\left((E, \rho)\right.$ is the space denoted by $P_{l_{2}}\left(E_{n}\right)$ in $\left.[2, \mathrm{p} .31].\right)$ It is easy to verify that $(E, \rho)$ is a reflexive Banach space and that the dual space of $(E, \rho)$ may be identified with $\left(E^{\prime}, \rho^{\prime}\right)$, where $E^{\prime}$ is the subspace of $\Pi E_{n}^{\prime}(n \in N)$ consisting of all $y=\left(y_{1}, y_{2}, \ldots\right)$ such that

$$
\rho^{\prime}(y)=\left(\sum_{n=1}^{\infty}\left[\rho_{n}^{\prime}\left(y_{n}\right)\right]^{2}\right)^{1 / 2}<\infty ;
$$

the pairing between $E$ and $E^{\prime}$ is given by $\langle x, y\rangle=\sum_{n=1}^{\infty}\left\langle x_{n}, y_{n}\right\rangle$.

Now let $r<1$ be an element of $R$ and let $n$ be in $N$. Pick $p_{k}$ such that $r n^{1 / p_{k}}$ $<1$. For each $j$ in $N$ let $e_{j}$ be the $K$-valued sequence which has the entry 1 in the $j$ th place and zeroes elsewhere. Then each $e_{j}$ belongs to $E_{k}$ and to $E_{k}^{\prime}$. For each $i$ with $1 \leq i \leq n$ define $x_{i}$ in $E_{k}$ by

$$
x_{i}=i^{-\left(1 / p_{k}\right)}\left(e_{1}+\cdots+e_{i}\right) .
$$

Then $\rho_{k}\left(x_{i}\right)=1, \rho_{k}^{\prime}\left(e_{j}\right)=1$ for $1 \leq i, j \leq n ; r<\left\langle x_{i}, e_{j}\right\rangle$ if $1 \leq j \leq i \leq n$; and $0=$ $\left\langle x_{i}, e_{j}\right\rangle$ if $1 \leq i<j \leq n$. Since $\left(E_{k}, \rho_{k}\right)$ and $\left(E_{k}^{\prime}, \rho_{k}^{\prime}\right)$ are embedded (as paired spaces $)$ in $(E, \rho)$ and $\left(E^{\prime}, \rho^{\prime}\right)$ respectively, it follows that $(E, \rho)$ does not satisfy condition (iii) of Theorem 8.5.

The conditions of Theorem 8.5 are satisfied, however, by many normed spaces. Recall that a normed space $(E, \rho)$ is called uniformly convex (or uniformly rotund) ([1] or [2, p. 112]) if for each $0<\epsilon \leq 2$ there exists $\delta(\epsilon)>0$ such that $\rho(x) \leq 1$, $\rho(y) \leq 1$ and $\rho(x-y)>\epsilon$ imply $\rho(x+y) \leq 2(1-\delta(\epsilon))$. It is easy to see that if $(E, \rho)$ is uniformly convex then $(\hat{E}, \hat{\rho})$ is also uniformly convex using the same function $\delta(\epsilon)$. Since complete uniformly convex spaces are reflexive ([7] and [8]), it follows that if $* \| \pi$ is $\aleph_{1}$-saturated and if $(E, \rho)$ is uniformly convex, then the conditions of Theorem 8.5 are satisfied. A direct proof of this fact is given next.

Theorem 8.7. Assume $* \mathbb{M}$ is $\boldsymbol{\aleph}_{1}$-saturated. If $(E, \rho)$ is a uniformly convex normed space in $\mathbb{M}$, then $(\hat{E}, \hat{\rho})$ is reflexive and the dual space of $(\hat{E}, \hat{\rho})$ is $\left(\widehat{E^{\prime}}, \widehat{\rho^{\prime}}\right)$. 
Proof. Since $(E, \rho)$ is uniformly convex, there exists $\delta=\delta(1 / 2)$ such that $\rho(x) \leq$ $1, \rho(y) \leq 1$ and $\rho(x-y)>1 / 2$ imply $\rho(x+y) \leq 2(1-\delta)$ for each $x, y$ in $E$. Let $r=$ $\max (1 / 2,1-\delta)<1$. Assume that $(E, \rho)$ is a real normed space: we will prove that condition (iii) of Theorem 8.5 holds for this $r$ and $n=2$. If not, then there exist $x_{1}$, $x_{2}$ in $E$ and $y_{1}, y_{2}$ in $E^{\prime}$ such that $\rho\left(x_{i}\right)=\rho\left(y_{j}\right)=1$ for $1 \leq i, j \leq 2 ; r<\left\langle x_{i}, y_{j}\right\rangle$ for $1 \leq j<i \leq 2$; and $0=\left\langle x_{1}, y_{2}\right\rangle$. Then $\left.\rho\left(x_{2}-x_{1}\right) \geq\left\langle x_{2}-x_{1}, y_{2}\right\rangle\right\rangle r \geq 1 / 2$, but $\rho\left(x_{1}+x_{2}\right) \geq\left\langle x_{1}+x_{2}, y_{1}\right\rangle>2 r \geq 2(1-\delta)$, which is a contradiction. The result now follows from Theorem 8.5.

The proof when $(E, \rho)$ is a complex normed space is similar, and uses the fact that $\rho(x) \geq \operatorname{Re}\langle x, y\rangle$ holds for any $x$ in $E$ and $y$ in $E^{\prime}$.

Finally, we note that J. A. Clarkson [1] has shown that $l_{p}$ and $L_{p}([0,1])$ are uniformly convex for $1<p<\infty$. Thus if $* M$ is $\aleph_{1}$-saturated, then the dual space of $\hat{l}_{p}$ is $\hat{l}_{q}$ and the dual space of $\hat{L}_{p}([0,1])$ is $\hat{L}_{q}([0,1])$, where $1<p<\infty$ and $q$ is defined by $1 / p+1 / q=1$.

Added in proof. Since submitting this paper for publication the authors discovered that Theorems 6.1 and 6.2 are contained in J. J. M. Chadwick and R. W. Cross, A characterization of pre-near-standardness in locally covvex linear topological spaces, Bull. Austral. Math. Soc. 6 (1972), 107-115; and also in L. Young, "Functional analysis-a nonstandard treatment with semifields," in W. A. J. Luxemburg and A. Robinson (Editors), Contributions to nonstandard analysis, North-Holland, Amsterdam, 1972.

\section{REFERENCES}

1. James A. Clarkson, Uniformly convex spaces, Trans. Amer. Math. Soc. 40 (1936), 396-414.

2. M. M. Day, Normed linear spaces, Ergebnisse der Mathematik und ihrer Grenzgebiete, Neue Folge, Heft 21. Reihe: Reelle Funktionen, Springer-Verlag, Berlin, 1958. MR 20 \#1187.

3. C. Ward Henson, The nonstandard hulls of a uniform space, Pacific J. Math. (to appear).

4. R. C. James, Characterizations of reflexivity, Studia Math. 23 (1963/64), 205-216. MR $30 \# 431$.

5. J. L. Kelley and I. Namioka, Linear topological spaces, University Series in Higher Math., Van Nostrand, Princeton, N. J., 1963. MR 29 \#3851.

6. W. A. J. Luxemburg, A general theory of monads, Internat. Sympos. Applications of Model Theory to Algebra, Analysis, and Probability (Pasadena, Calif., 1967), Holt, Rinehart and Winston, New York, 1969, pp. 18-86. MR 39 \#6244.

7. D. Milman, On some criteria for the regularity of spaces of the type B, C. R. (Dokl.) Acad. Sci. USSR 20 (1938), 243-246.

8. B. J. Pettis, A proof that every uniformly convex space is reflexive, Duke Math J. 5 (1939), 249-253.

9. Abraham Robinson, Non-standard analysis, North-Holland, Amsterdam, 1966. MR $34 \# 5680$. 